HD-TVP-94-16

\title{
How sharp is the chiral crossover phenomenon for realistic meson masses?
}

\author{
Hildegard Meyer-Ortmanns* \\ and \\ Bernd-Jochen Schaefer ${ }^{\dagger}$ \\ Institut für Theoret. Physik, \\ Universität Heidelberg \\ Philosophenweg 19 \\ D-69120 Heidelberg, F.R.G.
}

\begin{abstract}
The mass dependence of the chiral phase transition is studied in the linear $S U(3) \times S U(3)$ sigma-model to leading order in a $1 / N_{f}$-expansion, $N_{f}$ denoting the number of flavours. For realistic meson masses we find a smooth crossover between $T \sim 181.5$ to $192.6[\mathrm{MeV}]$. The crossover looks more rapid in the light quark condensate than in thermodynamic quantities like the energy and entropy densities. The change in the light quark condensate in this temperature interval is $\sim 50 \%$ of the zero-temperature condensate value, while the entropy density increases by $(5.5 \pm 0.8) \cdot 10^{-3}\left[\mathrm{GeV}^{3}\right]$. Since the numerical error is particularly large in this region, we cannot rule out a finite latent heat smaller than $0.2\left[\mathrm{GeV} / \mathrm{fm}^{3}\right]$. The chiral transition is washed out for an average pseudoscalar meson octet mass of 203 [MeV]. This gives an upper bound on the first-order transition region in the meson mass parameter space. The corresponding ratio of critical to realistic light current quark masses $m_{u, d}^{c r i t} / m_{u, d}$ is estimated as $0.26 \pm 0.08$. This result is by an order of magnitude larger than the corresponding mean-field value. Therefore the realistic quark/meson masses seem to lie less deeply in the crossover region than it is suggested by a mean-field calculation.
\end{abstract}

*e-mail address: ort@dhdmpi5.bitnet

$\dagger$ e-mail address: schaefer@hybrid.tphys.uni-heidelberg.de 


\section{Introduction}

Spontaneous symmetry breaking is frequently used as an ansatz to explain the driving force of finite temperature phase transitions in QCD. The symmetries refer to certain limiting cases of the QCD-Lagrangian. In the limit of infinite quark masses and in the case of three colours finite temperature QCD is invariant under $Z(3)$ transformations $(Z(3)$ is the center of $S U(3))$. The spontaneous breaking of $Z(3)$ is made responsible for the phase transition from the confinement phase at low temperatures to the deconfinement phase at high temperatures. In the other extreme case of $N_{f}$ vanishing current quark masses QCD is invariant under $S U\left(N_{f}\right) \times S U\left(N_{f}\right)$ chiral transformations. The restoration of the spontaneously broken chiral symmetry at high temperatures is said to drive the chiral phase transition of QCD. For the chiral limit the renormalization group analysis of Pisarski and Wilczek [1] serves as a guideline for conjectures about the order of the chiral phase transition. Similar studies have been performed by Svetitsky and Yaffe [2] in the other extreme case of pure gauge theory or infinite quark masses.

In reality the current quark masses are neither infinite nor zero. The masses of the charm, bottom and top quarks are large compared to the scale of the critical temperature $T_{c}$ of QCD, which lies between 150 and $250[\mathrm{MeV}]$. Thus it seems to be justified to treat them as infinite in thermodynamic investigations. The (renormalization group invariant current) masses of the up and down quarks are small compared to $T_{c}, m_{u}=7.6 \pm 2.2[\mathrm{MeV}], m_{d}=13.3 \pm 3.9[\mathrm{MeV}]$ for $\Lambda=100[\mathrm{MeV}]$, where $\Lambda$ refers to the $\overline{M S}$ scheme with 3 flavours [3]. At a first glance it seems to be well justified to set them simply to zero, although more careful investigations in the framework of chiral perturbation theory give us a warning to neglect their influence on the chiral transition [4]. A particular role is played by the strange quark. Its mass $\left(m_{s} \sim 205 \pm 50[\mathrm{MeV}]\right)$ is neither small nor large, but just of the order of the scale which is set by $T_{c}$. To get an idea about the influence of finite quark masses on the phase structure of QCD it is instructive to consult statistical physics. The phase transition from a liquid to a gas is of first order below some critical value of the pressure. For a critical value of the pressure it becomes of second order. Above the critical strength it turns into a smooth crossover between the liquid and the gas phase. Similar effects are known from ferromagnets under the influence of an external magnetic field. In an $O(N)$-ferromagnet an arbitrarily small magnetic field is sufficient to turn the second order transition with an infinite correlation length into a crossover without a diverging correlation length. In close analogy to our subsequent considerations we should also mention the three-dimensional $Z(3)$-Potts 
model. The spin variables of this model can take three values at each lattice site. For a vanishing external magnetic field the Potts model is known to have a first order phase transition at finite temperature. For a critical field strength it becomes of second order and disappears for even stronger external fields.

The conjugate variables are the pressure and the specific volume in the liquid/gas system, the external magnetic field and the magnetization for the ferromagnet. The analogous pair in QCD are the current quark masses and the quark condensates as the associated order parameters for the chiral transition (on the quark level). On the mesonic level we have external fields, which can be related to the quark masses, and mesonic condensates. The comparison between the statistical systems and QCD goes beyond a formal analogy. In an $S U(3)$-lattice gauge theory it has been shown by DeGrand and DeTar [5] and Banks and Ukawa [6] that dynamical fermions on the QCD level induce an external field coupled linearly to a spin field in an effective $Z(3)$-spin model. The spin model has been derived from QCD in a strong coupling expansion at high temperatures. The external field strength was expressed in terms of the hopping parameter of the lattice-QCD Lagrangian.

In this paper, we consider the $S U(3) \times S U(3)$ linear sigma-model as an effective model for chiral symmetry restoration. We include two external fields to account for the finite quark masses on the mesonic level. The action is constructed in terms of the chiral order parameter field, which is a matrix of mesonic condensates. In the special case of one external field and one mesonic condensate, the action of the sigma-model takes the form of Landau's free energy functional for a liquid/gas system (cf. section 5). Thus we expect the same qualitative features as we know from the liquid/gas system. Beyond certain critical values of the meson masses the chiral transition should be washed out and turn into a crossover phenomenon with smooth changes in the condensates, energy density and entropy density. Therefore the main question is, as to whether the realistic quark masses are too large for the chiral transition and too small for the deconfinement transition to persist.

Numerous numerical simulations in lattice QCD have investigated the phase structure of QCD. Results on the mass dependence of the finite temperature transitions were summarized in the famous Columbia-plot [7], cf. Fig. 1. Indicated are the presence or absence of QCD transitions as a function of the bare quark masses $m_{s} \cdot a$ and $m_{u, d} \cdot a$ in lattice units, $a$ denotes the lattice constant. The solid line stands for second order transitions, the shaded areas enclose mass values leading to first order 
Figure 1: Columbia plot [7]. Generic phase diagram, partly conjectural, for 2 and 3 flavours. Solid lines indicate supposed 2nd order transitions, shaded areas 1st order transitions. Solid circles correspond to mass parameters, which lead to 1st order transitions, triangles to mass parameters, which lead to crossover phenomena. The open circle locates the suggested physical mass point.

transitions. Solid circles correspond to mass values leading to 1st order transitions, e.g. the point with 3 degenerate bare quark masses $m_{u} a=m_{d} a=m_{s} a=0.025$. Triangles indicate mass combinations which lead to a crossover phenomenon. In particular, the mass point is included with two light $\left(m_{u} a=m_{d} a=0.025\right)$ and one heavier flavour $\left(m_{s} a=0.1\right)$. This mass point comes closest to the realistic quark masses with a ratio of $m_{s} / m_{u, d} \sim 20$. The ratio between the critical quark mass $m_{u}^{\text {crit }}$ which lies on the first order transition boundary to the realistic quark mass $m_{u}$ is about 0.5 if the ratio $m_{u}^{c r i t} a / m_{s}^{c r i t} a=m_{u} a / m_{s} a$ is kept fixed. Fig. 1 suggests that the masspoint with realistic quark masses is not too far from the second order transition line, although the precise values of the critical strange quark masses, where the first order transitions turn into second order transitions, are still an open question.

Thus the conclusion from the lattice is that there is no true chiral phase transition for experimental quark masses. Depending on the degree of smoothness of the chiral transition this conclusion can have far reaching consequences in view of measurable effects in heavy-ion collisions. Therefore we spend some comments on the reliability of the lattice result.

Monte Carlo results from lattice calculations are in general affected by artifacts due to the finite IR-cutoff (i.e. the finite volume), the UV-cutoff (i.e. the finite lattice constant) and finite bare quark masses (it is unavoidable to extrapolate to 
the chiral limit). Applied to the masspoint with two light and one heavier flavour ( $\left.m_{u, d} a=0.025, m_{s} a=0.1\right)$ of the Columbia plot [7] there are some warnings which should be kept in mind. The lattice extension in imaginary time direction was chosen as $N_{\tau}=4$. Even without dynamical fermions $N_{\tau}$ should be larger than 10 to find a critical bare coupling close to the continuum limit. One manifestation of the vicinity to the strong coupling regime are the masses of flavour partners which should be degenerate in the continuum limit. The ratio of two $K$-meson masses is still about 2 [7]. The effect of heavier flavour partners on the order of the chiral transition is difficult to control.

Another subtle effect of the finite UV-cutoff is a change in the effective symmetry as a function of the bare coupling if staggered fermions are used [8]. For four continuum flavours the chiral transition is of 2nd order in the strong coupling limit, but of 1st order close to the continuum region. For two flavours the critical indices of the second order transition change between the strong and weak coupling region [9]. For three flavours one can speculate, whether the crossover itself is a strong coupling feature rather than continuum physics.

A further obstacle in the staggered fermion formulation applies in particular to the case of three flavours. The effective fermionic action which projects on three flavours must be regarded as a prescription rather than being derived from the staggered fermion action [7]. A derivation could only lead to integer multiples of four continuum flavours in the effective action. The error due to this prescription seems to be difficult to control.

A bulk "chiral" transition has been identified for 8 flavours [10] (for bulk transitions the transition itself is a mere lattice artifact). Precursors of this bulk transition for a smaller number of flavours can be superimposed on the finite $T$-chiral transition and influence its strength.

If the bare coupling lies in the strong coupling regime, the conversion of bare masses in lattice units into masses in $[\mathrm{MeV}]$ is not unique. Last but not least it is not clear whether the ratio of $m_{u, d} a / m_{s} a$ is unaffected by multiplicative renormalizations (it may be that $m_{u, d}^{r e n} / m_{s}^{r e n} \neq m_{u, d} a / m_{s} a$ ). Hence the very location of the physical mass point in the $\left(m_{u, d} a, m_{s} a\right)$ diagram is questionable.

These warnings should suffice as arguments in favour of alternative approaches which include different approximations to study the phase structure of QCD.

In [11] a systematic study of the mass dependence of the chiral transition has 
been proposed in the framework of effective models for QCD. In a first paper [11] a bound on the first order transition region was given for the $S U(3)$-symmetric case. The loose bound on the (average) pseudoscalar octet mass was $100[\mathrm{MeV}]$ for a sigma meson mass between 600 and $950[\mathrm{MeV}]$. The qualitative conclusion was that realistic meson masses lie deeply in the crossover region. The results were obtained in a large $N_{f}$-expansion under the omission of $n \neq 0$-Matsubara frequencies.

In this paper we will give a more stringent bound on the maximal pseudoscalar octet mass, for which the transition is still of first order. The large $N_{f}$-expansion seems to be a good starting point for the $S U(3) \times S U(3)$ linear sigma-model, which reduces to an $O(18)$-model in a special case of two vanishing couplings, see section 3 below. Therefore we still use the large $N_{f}$-expansion, but include also the $n \neq 0$ Matsubara frequencies. The omission of the $n \neq 0$-modes seems to be justified only for high temperatures or in case of a second order phase transition. The distance (in mass parameter space) between experimental meson masses and critical meson masses, for which the first order chiral transition turns into a crossover phenomenon, is more than a quantity of academic interest. Its physical relevance can be seen as follows. Assume that the physical masses are not identical but very close to the critical mass values where the chiral transition is of second order. In such a case the chances are good to see some remnant in heavy-ion collisions of a diverging correlation length for critical mass values, i.e. a rather large correlation length for realistic masses.

One manifestation of a large correlation length has been supposed to be large clusters of charged or neutral pions which are aligned in isospin space [12]. The correlation volume of a cluster should be large enough that the number of emitted pions is sufficient for the detector to resolve the cluster structure. Intermittent behaviour has been proposed as another manifestation of a second order transition [13. In this case one would observe some kind of self-similarity phenomenon if the bin size of rapidity intervals is made finer and finer.

Also the vicinity to the first order transition region could leave observable effects if the crossover for realistic masses is still sharp enough. A sharp crossover is associated with a rapid change in the condensates and their induced masses, and/or a rapid change of entropy in a small temperature interval. Average transverse momentum distributions of charged particles could be flattened as a function of the multiplicity $d N / d y$ of final state particles in a given rapidity interval [14. Pronounced fluctuations in the particle multiplicities would show up if the crossover is strong enough to induce deflagration processes during the phase conversion [15]. True singularities of a first or second order transition will be anyway rounded in real experiments due 
to the finite volume.

It may be academic to ask as to whether chiral symmetry restoration in the infinite volume limit proceeds via a weakly first order transition or a smooth crossover phenomenon. It is certainly more sensible to pose the question in the following way: Is the gap in entropy densities in the transition/crossover region sufficient to induce multiplicity fluctuations in the observed pion yield, lying clearly above the statistical noise? In this paper, we therefore try to find a quantitative measure for how sharp the chiral crossover phenomenon is for realistic meson masses.

The paper is organized as follows. In section 2 we present the tree level parametrization of the $S U(3) \times S U(3)$-linear sigma-model at zero temperature. We give a prescription to translate the meson condensates and meson masses to quark condensates and current quark masses. In section 3 we summarize the essence of our approach. The mesonic self-interaction is treated to leading order in an expansion in the number of quark flavours $N_{f}$. On the mesonic level we have 9 scalar plus 9 pseudoscalar mesons. The $S U(3) \times S U(3)$ sigma-model reduces to an $O(18)$-model in a certain limit. Thus the leading order of a $1 / N$-expansion should be a good starting point. The thermodynamic effective potential is evaluated in a high-temperature expansion and in a fully numerical approach. The numerical approach is certainly more appropriate to the phase transition region. To discuss the mass dependence of the order of the chiral transition we distinguish three regions in mass parameter space $\left(m_{\pi}, m_{K}, \ldots\right)$ and $\left(m_{u, d}, m_{s}\right)$ : the chiral limit (section 4$)$, several mass points on the first order transition boundary, so-called critical mass points (section 5) and realistic meson masses (section 6), which come close to the experimental masses. In the chiral limit we fix the couplings of the tree level parametrization. The quantitative discrepancy between the high-temperature expansion and the fully numerical evaluation will be revealed. We calculate the barrier height between the coexisting minima of the effective potential as one measure for the strength of the first order transition.

Values of critical meson masses are determined in section 5 for three special cases: the $S U(3)$-symmetric case with finite, but degenerate pseudoscalar octet masses, the case with a realistic mass splitting between the masses of the pseudoscalar octet (the ratios of meson masses with and without strangeness are kept fixed to their experimental values), and the case of vanishing strange quark mass $m_{s}$. The critical meson masses are calculated both in a mean-field approximation and in the large $N_{f}$-expansion.

Section 6 deals with meson masses which are rather close to the experimental values. 
We describe the crossover phenomenon in the meson and quark condensates as a function of temperature. The crossover is also manifest in thermodynamic quantities like the energy density $\varepsilon$, the entropy density $s$, and the pressure $p$. We derive $\varepsilon, s$ and $p$ from the partition function of the sigma-model in a saddle-point approximation. Upper bounds on a finite latent heat during the chiral transition are predicted. The temperature dependence of effective masses which enter the calculation of the effective potential will be displayed. In section 7 we summarize our results and draw some conclusions in view of heavy-ion experiments.

\section{Tree level parametrization of the $S U(3) \times S U(3)$ linear sigma-model}

The Euclidean Lagrangian density of the $S U(3) \times S U(3)$-linear sigma-model is given as

$$
\begin{aligned}
\mathcal{L}_{\text {Eucl }} & =\frac{1}{2} \operatorname{Tr}\left(\partial_{\mu} M \partial_{\mu} M^{+}\right)-\frac{1}{2} \mu_{0}^{2} \operatorname{Tr} M M^{+}+g\left(\operatorname{det} M+\operatorname{det} M^{+}\right)+ \\
& +f_{1}\left(\operatorname{Tr} M M^{+}\right)^{2}+f_{2} \operatorname{Tr}\left(M M^{+}\right)^{2}-\varepsilon_{0} \sigma_{0}-\varepsilon_{8} \sigma_{8}
\end{aligned}
$$

where the $(3 \times 3)$-matrix field $M(x)$ is written as

$$
M=\frac{1}{\sqrt{2}} \sum_{\ell=0}^{8}\left(\sigma_{\ell}+i \pi_{\ell}\right) \lambda_{\ell}
$$

Here $\sigma_{\ell}$ and $\pi_{\ell}$ denote the nonets of scalar and pseudoscalar mesons, respectively,

$\lambda_{\ell}(\ell=1, \ldots, 8)$ are the Gell-Mann matrices, $\lambda_{0}=\sqrt{\frac{2}{3}} \cdot \operatorname{diag}(1,1,1)$. The chiral $S U(3) \times S U(3)$-symmetry is explicitly broken by the term $\left(-\varepsilon_{0} \sigma_{0}-\varepsilon_{8} \sigma_{8}\right)$, which is linear in the external fields $\varepsilon_{0}, \varepsilon_{8}$. A non-vanishing value of $\varepsilon_{0}$ gives a common mass value to the octet of pseudoscalar Goldstone bosons $m_{\pi}, m_{k}, m_{\eta}$. When also $\varepsilon_{8} \neq 0$, it can be adjusted such that it leads to a realistic mass splitting inside the (pseudo)scalar meson octets.

The Lagrangian (11) appears as a natural candidate for an effective model, which is designed to describe the phenomenon of chiral symmetry restoration. The action $S=\int d^{3} x d \tau \mathcal{L}(x)$ with $\mathcal{L}$ of Eq. (1) may be regarded as an effective action for QCD, constructed in terms of a chiral order parameter field $M$ for the chiral transition. As order parameters for the chiral transition we choose the meson condensates $\left\langle\sigma_{0}\right\rangle$ and $\left\langle\sigma_{8}\right\rangle$. The expectation value of $M$ is then parametrized in terms of $\left\langle\sigma_{0}\right\rangle,\left\langle\sigma_{8}\right\rangle$ according to

$$
\langle M\rangle=\operatorname{diag} \frac{1}{\sqrt{3}}\left[\left\langle\sigma_{0}\right\rangle+\frac{1}{\sqrt{2}}\left\langle\sigma_{8}\right\rangle,\left\langle\sigma_{0}\right\rangle+\frac{1}{\sqrt{2}}\left\langle\sigma_{8}\right\rangle,\left\langle\sigma_{0}\right\rangle-\sqrt{2}\left\langle\sigma_{8}\right\rangle\right] .
$$


The construction of an action $S$ in terms of an order parameter field is a concept in close analogy to Landau's free energy functional $\mathcal{F}$ in terms of an order parameter field, $\mathcal{F}$ coincides with $S$ in the mean-field approximation. Quartic terms in $M$ have to be introduced in Eq. (1) to allow for the possibility of spontaneous symmetry breaking. For $g=0=\varepsilon_{0}=\varepsilon_{8}$ the Lagrangian is still invariant under $U(3) \times U(3)$ transformations. For $N_{f} \geq 3$ there are two independent quartic terms, parametrized with coefficients $f_{1}$ and $f_{2}$. To account for a realistic $\left(\eta, \eta^{\prime}\right)$-mass splitting, a detterm with an "instanton"-coupling $g$ has to be included as well. It reduces the symmetry of $\mathcal{L}$ to $S U(3) \times S U(3)$ if $\varepsilon_{0}=0=\varepsilon_{8}$. Finally, the external field terms which are linear in $M$ are the most simple choice for an explicit symmetry breaking accounting for the small, but finite masses of the Goldstone octet $\left(m_{\pi}, m_{K}, m_{\eta}\right)$. Thus one arrives at the $S U(3) \times S U(3)$ linear sigma-model in a natural way if one is interested in the limited aspect of chiral symmetry restoration.

It remains to fix the Lagrangian parameters $\mu_{0}^{2}, f_{1}, f_{2}, g, \varepsilon_{0}, \varepsilon_{8}$ from an experimental input. The choice of the input and the way of parametrization are in no way unique, cf. [16, 17, 11]. Since the pseudoscalar meson masses are experimentally well known, $m_{\pi}, m_{k}, m_{\eta}, m_{\eta^{\prime}}$ and $f_{\pi}$ have been used in [11] to fix $\mu_{0}^{2}, f_{1}, f_{2}, g, \varepsilon_{0}$ and $\varepsilon_{8}$. In addition, the mass of the $\sigma^{\prime}$-meson has been treated as input parameter and varied between 600 and $950[\mathrm{MeV}]$ to solve for $\mu_{0}^{2}$ and $f_{1}$, which occur in the (pseudo)scalar meson masses in the combination $\left(-\mu_{0}^{2}+4 f_{1}\left(\sigma_{0}^{2}+\sigma_{8}^{2}\right)\right)$. It is worth noting that the order parameters $\left\langle\sigma_{0}\right\rangle,\left\langle\sigma_{8}\right\rangle$ can be determined without knowing $\mu_{0}^{2}$ and $f_{1}$ separately. The equations for $\mu_{0}^{2}, f_{1}, f_{2}, g, \varepsilon_{0}, \varepsilon_{8}$ do not admit solutions for an arbitrary choice of $m_{\pi}, m_{k}, m_{\eta}, m_{\eta^{\prime}}, f_{\pi}$ and $m_{\sigma}$. This was the reason why the input masses which have been actually used as input in [11] were slightly deviating from the experimental values if $m_{\sigma_{\eta^{\prime}}}$ was chosen as 600 or $950[\mathrm{MeV}]$. In [18] the experimental values could be used for $m_{\pi}, m_{k}, m_{\eta}, m_{\eta^{\prime}}, f_{\pi}$ on the price that $m_{\sigma_{\eta^{\prime}}}$ was used as input with $1400[\mathrm{MeV}]$.

In this paper, our interest goes beyond the point with (almost) experimental pseudoscalar meson masses. Naturally, the question arises why we are not satisfied with a parametrization that reproduces the experimental values for the (pseudo)scalar meson masses, but want to tune some parameters to unphysical, unrealistic mass values as well. There are two reasons for that. The first one is to check how stable our results about the phase structure are under a slight change of the mass input. The second one is to get a quantitative measure for the distance (in mass parameter space) between the realistic and the critical meson masses, cf. section 5 below. As we focus on the aspect of the mass sensitivity, we have to find a 
prescription how to tune the masses in the high-dimensional meson mass parameter space. On the quark level the mass parameter space is only two-dimensional, the two parameters are $m_{u, d}$ and $m_{s}$. Not only the parametrization, but also the tuning in the space of meson masses is by far not unique. The idea now is to parametrize the (pseudo)scalar meson masses by two parameters like the quark masses, the external fields $\varepsilon_{0}$ and $\varepsilon_{8}$.

A relation between $m_{u, d}, m_{s}$ and $\varepsilon_{0}, \varepsilon_{8}$ is obtained by identifying terms of the Lagrangian on the mesonic and on the quark level which transform identically under $S U(3) \times S U(3)$. We have

$$
\begin{aligned}
\left(-\varepsilon_{0} \sigma_{0}-\varepsilon_{8} \sigma_{8}\right) & \text { on the mesonic level, } \\
+\left(m_{u} \bar{u} u+m_{d} \bar{d} d+m_{s} \bar{s} s\right) & \text { on the quark level. }
\end{aligned}
$$

Thus we find

$$
\begin{aligned}
-\varepsilon_{0} & =\alpha\left(2 \hat{m}+m_{s}\right) \\
-\varepsilon_{8} & =\beta\left(\hat{m}-m_{s}\right) .
\end{aligned}
$$

Here $\alpha$ and $\beta$ are constants. They can be fixed from the known values of $\varepsilon_{0}, \varepsilon_{8}, m_{u, d}$ and $m_{s}$ under realistic conditions. Realistic meson masses are obtained for $\varepsilon_{0}=$ $0.0265[\mathrm{GeV}]^{3}, \varepsilon_{8}=-0.0345[\mathrm{GeV}]^{3}$, see below. The values for "realistic" current quark masses are taken from [19], $\hat{m} \equiv\left(m_{u}+m_{d}\right) / 2=11.25 \pm 1.45[\mathrm{MeV}], m_{s}=$ $205 \pm 50[\mathrm{MeV}]$. For $\alpha$ and $\beta$ we then obtain

$$
\alpha=-0.1164[\mathrm{GeV}]^{2}, \quad \beta=-0.1780[\mathrm{GeV}]^{2} .
$$

Thus a variation in $\left(\varepsilon_{0}, \varepsilon_{8}\right)$ can be mapped onto a variation of $\left(\hat{m}, m_{s}\right)$.

Next we have to find a mapping between $\left(\varepsilon_{0}, \varepsilon_{8}\right)$ and the (pseudo)scalar meson masses. As it is possible to explain the variety of (pseudo)scalar meson masses on the basis of two quark masses $m_{u, d}$ and $m_{s}$, it should be similarly possible to reach any point in an $\left(m_{\pi}, m_{K, \ldots}\right)$-diagram by a variation of $\varepsilon_{0}$ and $\varepsilon_{8}$. Thus we start with the parametrization of the chiral limit $\mu_{0}^{2}, f_{1}, f_{2}, g, \varepsilon_{0}=0, \varepsilon_{8}=0$, keep the couplings $\mu_{0}^{2}, f_{1}, f_{2}, g$ fixed and switch on $\varepsilon_{0} \neq 0, \varepsilon_{8} \neq 0$. The $S U(3)$-symmetric case with finite, but degenerate pseudoscalar meson masses is obtained for $\varepsilon_{0} \neq 0, \varepsilon_{8}=0$. The mass point with realistic meson masses which come close to their experimental values, is obtained for $\epsilon_{0}=0.0265[\mathrm{GeV}]^{3}, \varepsilon_{8}=-0.0345[\mathrm{GeV}]^{3}$. (In principle, there is no obstacle to further optimize the values of $\varepsilon_{0}, \varepsilon_{8}$ such that they do reproduce the experimental mass values). For the chiral values $\mu_{0}^{2}, f_{1}, f_{2}, g$ and a certain choice 
for $\varepsilon_{0}$ and $\varepsilon_{8}$ we first determine $\left\langle\sigma_{0}\right\rangle$ and $\left\langle\sigma_{8}\right\rangle$, the condensates at zero temperature, as zeroes of (8),(9) in $\sigma_{0}$ and $\sigma_{8}$

$$
\begin{array}{r}
\varepsilon_{0}+\mu_{0}^{2} \sigma_{0}-\frac{g}{\sqrt{3}}\left(2 \sigma_{0}^{2}-\sigma_{8}^{2}\right)+\frac{2 \sqrt{2}}{3} f_{2} \sigma_{8}^{3}-4\left(f_{1}+\frac{f_{2}}{3}\right) \sigma_{0}^{3}-4\left(f_{1}+f_{2}\right) \sigma_{0} \sigma_{8}^{2}=0 \\
\varepsilon_{8}+\mu_{0}^{2} \sigma_{8}+\sqrt{\frac{2}{3}} g\left(\sigma_{8}^{2}+\sqrt{2} \sigma_{0} \sigma_{8}\right)+2 \sqrt{2} f_{2} \sigma_{0} \sigma_{8}^{2}-4\left(f_{1}+\frac{f_{2}}{2}\right) \sigma_{8}^{3}-4\left(f_{1}+f_{2}\right) \sigma_{0}^{2} \sigma_{8}=0 .
\end{array}
$$

Eqs. (8,9) are the equations of motion for constant background fields $\sigma_{0}, \sigma_{8}$. The pseudoscalar meson masses are then given as

$$
\begin{aligned}
m_{\pi}^{2} & =-\mu_{0}^{2}+\left(4 f_{1}+\frac{4}{3} f_{2}\right) \sigma_{0}^{2}+\left(4 f_{1}+\frac{2}{3} f_{2}\right) \sigma_{8}^{2}+\frac{4}{3} \sqrt{2} f_{2} \sigma_{0} \sigma_{8}+\frac{2 g}{\sqrt{3}} \sigma_{0}-2 \sqrt{\frac{2}{3}} g \sigma_{8} \\
m_{k}^{2} & =-\mu_{0}^{2}+\left(4 f_{1}+\frac{4}{3} f_{2}\right) \sigma_{0}^{2}+\left(4 f_{1}+\frac{14}{3} f_{2}\right) \sigma_{8}^{2}-\frac{2}{3} \sqrt{2} f_{2} \sigma_{0} \sigma_{8}+\frac{2}{\sqrt{3}} g \sigma_{0}+\sqrt{\frac{2}{3}} g \sigma_{8} \\
m_{\eta_{00}}^{2} & =-\mu_{0}^{2}+\left(4 f_{1}+\frac{4}{3} f_{2}\right) \sigma_{0}^{2}+\left(4 f_{1}+\frac{4}{3} f_{2}\right) \sigma_{8}^{2}-\frac{4}{\sqrt{3}} g \sigma_{0} \\
m_{\eta_{88}}^{2} & =-\mu_{0}^{2}+\left(4 f_{1}+\frac{4}{3} f_{2}\right) \sigma_{0}^{2}+\left(4 f_{1}+2 f_{2}\right) \sigma_{8}^{2}-\frac{4}{3} \sqrt{2} f_{2} \sigma_{0} \sigma_{8}+\frac{2}{\sqrt{3}} g \sigma_{0}+2 \sqrt{\frac{2}{3}} g \sigma_{8} \\
m_{\eta_{08}}^{2} & =\sigma_{8}\left[\frac{8}{3} f_{2} \sigma_{0}-\frac{2}{3} \sqrt{2} f_{2} \sigma_{8}+\frac{2}{\sqrt{3}} g\right] \\
m_{\eta}^{2} & =\frac{1}{2}\left(m_{\eta_{00}}^{2}+m_{\eta_{88}}^{2}-\sqrt{\left(m_{\eta_{00}}^{2}-m_{\eta_{88}}^{2}\right)^{2}+4\left(m_{\eta_{08}}^{2}\right)^{2}}\right) \\
m_{\eta^{\prime}}^{2} & =\frac{1}{2}\left(m_{\eta_{00}}^{2}+m_{\eta_{88}}^{2}+\sqrt{\left(m_{\eta_{00}}^{2}-m_{\eta_{88}}^{2}\right)^{2}+4\left(m_{\eta_{08}}^{2}\right)^{2}}\right) .
\end{aligned}
$$

The scalar meson masses follow from

$$
\begin{aligned}
& m_{\sigma_{\pi}}^{2}=-\mu_{0}^{2}+\left(4 f_{1}+4 f_{2}\right) \sigma_{0}^{2}+\left(4 f_{1}+2 f_{2}\right) \sigma_{8}^{2}+\frac{8}{\sqrt{2}} f_{2} \sigma_{0} \sigma_{8}-\frac{2}{\sqrt{3}} g \sigma_{0}+2 \sqrt{\frac{2}{3}} g \sigma_{8} \\
& m_{\sigma_{k}}^{2}=-\mu_{0}^{2}+\left(4 f_{1}+4 f_{2}\right) \sigma_{0}^{2}+\left(4 f_{1}+2 f_{2}\right) \sigma_{8}^{2}-\frac{4}{\sqrt{2}} f_{2} \sigma_{0} \sigma_{8}-\frac{2}{\sqrt{3}} g \sigma_{0}-\sqrt{\frac{2}{3}} g \sigma_{8} \\
& m_{\sigma_{00}}^{2}=-\mu_{0}^{2}+\left(12 f_{1}+4 f_{2}\right) \sigma_{0}^{2}+4\left(f_{1}+f_{2}\right) \sigma^{8}+\frac{4 g}{\sqrt{3}} \sigma_{0} \\
& m_{\sigma_{88}}^{2}=-\mu_{0}^{2}+4\left(f_{1}+f_{2}\right) \sigma_{0}^{2}+6\left(2 f_{1}+f_{2}\right) \sigma_{8}^{2}-4 \cdot \sqrt{2} f_{2} \sigma_{0} \sigma_{8}-\frac{2 g}{\sqrt{3}} \sigma_{0}-2 \sqrt{\frac{2}{3}} g \sigma_{8} \\
& m_{\sigma_{08}}^{2}=\sigma_{8}\left[8\left(f_{1}+f_{2}\right) \sigma_{0}-\frac{4}{\sqrt{2}} f_{2} \sigma_{8}-\frac{2}{\sqrt{3}} g\right] \\
& m_{\sigma_{\eta}}^{2}=\frac{1}{2}\left(m_{\sigma_{00}}^{2}+m_{\sigma_{88}}^{2}+\sqrt{\left(m_{\sigma_{00}}^{2}-m_{\sigma_{88}}^{2}\right)^{2}+4\left(m_{\sigma_{08}}^{2}\right)^{2}}\right) \\
& m_{\sigma_{\eta^{\prime}}}^{2}=\frac{1}{2}\left(m_{\sigma_{00}}^{2}+m_{\sigma_{88}}^{2}-\sqrt{\left(m_{\sigma_{00}}^{2}-m_{\sigma_{88}}^{2}\right)^{2}+4\left(m_{\sigma_{08}}^{2}\right)^{2}}\right),
\end{aligned}
$$


where $\sigma_{0}, \sigma_{8}$ should be replaced by $\left\langle\sigma_{0}\right\rangle,\left\langle\sigma_{8}\right\rangle$, respectively, everywhere in Eqs. $(10,11)$ for physical masses. The resulting masses together with the input parameters are listed in Table 1 of section 6.

To facilitate a comparison with other work on the chiral phase transition, it remains to translate the results for the meson condensates to the quark level. If we identify terms in the Lagrangians on the quark level and on the mesonic level (cf. Eqs. (4) and (5)), we find

$$
\begin{aligned}
\frac{1}{3}\left(2 \hat{m}+m_{s}\right)(2 \bar{q} q+\bar{s} s) & =-\varepsilon_{0} \sigma_{0} \\
\frac{2}{3}\left(\hat{m}-m_{s}\right)(\bar{q} q-\bar{s} s) & =-\varepsilon_{8} \sigma_{8}
\end{aligned}
$$

with $\bar{q} q=\frac{1}{2}(\bar{u} u+\bar{d} d)$, leading to

$$
\begin{aligned}
\langle\bar{q} q\rangle & =\frac{-\varepsilon_{0}}{2 \hat{m}+m_{s}}\left\langle\sigma_{0}\right\rangle+\frac{-\varepsilon_{8}}{2\left(\hat{m}-m_{s}\right)}\left\langle\sigma_{8}\right\rangle \\
\langle\bar{s} s\rangle & =\frac{-\varepsilon_{0}}{2 \hat{m}+m_{s}}\left\langle\sigma_{0}\right\rangle+\frac{+\varepsilon_{8}}{\hat{m}-m_{s}}\left\langle\sigma_{8}\right\rangle .
\end{aligned}
$$

The coefficients are just proportional to $\alpha$ and $\pm \beta$, as a comparison with Eq. (6) shows, $\alpha$ and $\beta$ have been determined in Eq. (7). Later we take the relations (14) as temperature independent and substitute $\left\langle\sigma_{0}\right\rangle(T),\left\langle\sigma_{8}\right\rangle(T)$ for the corresponding condensates at zero temperature. This assumption is consistent with our approach. We also treat the couplings $\mu_{0}^{2}, f_{1}, f_{2}, g$ of the Lagrangian as temperature independent. Therefore the symmetry of $\mathcal{L}$ remains unchanged under an increase of $T$. The

symmetry was on the basis of the identification which has led to Eqs. (14). (The assumption of temperature independent couplings may not be justified in the vicinity of the transition region.) In the next section we will outline how to calculate $\left\langle\sigma_{0}\right\rangle(T),\left\langle\sigma_{8}\right\rangle(T)$.

\section{Large $N_{f}$-expansion}

In an earlier calculation the linear $S U(3) \times S U(3)$ sigma-model has been considered in a mean-field approximation [20]. Recently, Gavin, Goksch and Pisarski [17] have tried to localize the first order transition boundary in an $\left(m_{u, d}, m_{s}\right)$-mass diagram in a mean-field calculation. The famous renormalization group analysis of Pisarski and Wilczek [1] applied to the linear sigma-model in the chiral limit. Frei and Patkós [21] were the first to apply a saddle-point approximation to the partition function of the sigma-model. Their investigations were also restricted to the chiral limit. MeyerOrtmanns, Patkós and Pirner [1]] have extended the approach of Frei and Patkós to 
finite meson masses. In [11] only the zero-Matsubara frequencies were kept. When the imaginary time dependence of the fields or, equivalently, the $n \neq 0$-Matsubara frequencies are dropped, it results in a dimensional reduction of the four-dimensional theory to an effective three-dimensional theory. In general, such a reduction can be justified in the high- $T$-limit or for an anticipated second order phase transition. In both cases the ratio of $\beta / \xi$ is negligible ( $\beta$ is the inverse temperature and $\xi$ denotes the largest correlation length of the system). The $n \neq 0$-Matsubara modes were included in [18]. In this paper, we follow the same approach as in [18], but extend the work to study the aspect of the mass sensitivity of the chiral transition. Further differences in details of [18] and the present paper will be mentioned below. In the following, we summarize the essence of the large- $N_{f}$-approach to make the paper self-contained.

The temperature-dependent order parameters are the meson condensates $\left\langle\sigma_{0}\right\rangle(T)$ and $\left\langle\sigma_{8}\right\rangle(T)$. They are related to the light and strange quark condensates according to Eqs. (14). The values of $\left\langle\sigma_{0}\right\rangle(T),\left\langle\sigma_{8}\right\rangle(T)$ are determined as the minima of an effective potential $\hat{U}_{e f f}\left(\sigma_{0}, \sigma_{8}\right)$. The effective potential is calculated as a constrained free energy density, i.e. the free energy density under the constraint that the average fields $\frac{1}{\beta V} \int_{0}^{\beta} d \tau \int d^{3} x \sigma_{0,8}(\vec{x}, \tau)$ take prescribed values $\bar{\sigma}_{0}$ and $\bar{\sigma}_{8}$, respectively, while the same averages over $\sigma_{\ell}, \ell=1, \ldots, 7$, and $\pi_{\ell}, \ell=0, \ldots, 8$ should vanish. The physical meaning is obvious. If one chooses $\bar{\sigma}_{0}, \bar{\sigma}_{8} \neq 0$ in the high-temperature chiral symmetric phase, the corresponding free energy is certainly not minimal for such a choice. We consider spontaneous symmetry breaking in two directions. Accordingly, we introduce two background fields $\bar{\sigma}_{0}, \bar{\sigma}_{8}$

$$
\begin{aligned}
& \sigma_{0}=\bar{\sigma}_{0}+\sigma_{0}^{\prime} \\
& \sigma_{8}=\bar{\sigma}_{8}+\sigma_{8}^{\prime},
\end{aligned}
$$

where $\sigma_{0}^{\prime}, \sigma_{8}^{\prime}$ denote the fluctuations around the background. Otherwise, $\sigma_{\ell}=\sigma_{\ell}^{\prime}$ for $\ell=1, \ldots, 7$ and $\pi_{\ell}=\pi_{\ell}^{\prime}$ for $\ell=0, \ldots, 8$. As a common notation for $\sigma_{\ell}^{\prime}, \pi_{\ell}^{\prime}$ we use $M_{\ell}^{\prime}=\sigma_{\ell}^{\prime}+i \pi_{\ell}^{\prime}$. The actual minima of $\hat{U}_{e f f}\left(\sigma_{0}, \sigma_{8}\right)$ (with $\sigma_{0}=\bar{\sigma}_{0}, \sigma_{8}=\bar{\sigma}_{8}$ ) are denoted as $\left\langle\sigma_{0}\right\rangle$ and $\left\langle\sigma_{8}\right\rangle$.

The Lagrangian is expanded in powers of $M_{\ell}^{\prime}$. The linear term in $M_{\ell}^{\prime}$ vanishes due to the $\delta$-constraint in the constrained free energy density. The quadratic term defines the masses $m_{Q}^{2}$ of the meson multiplets $\pi, K, \eta, \eta^{\prime}, \sigma_{\pi}, \sigma_{K}, \sigma_{\eta}, \sigma_{\eta^{\prime}}, Q=1, \ldots, 8$ labels the multiplets. The explicit formulas were given in Eqs. (10),(11). The two quartic terms are quadratized by introducing an auxiliary matrix field $\sum(x)$ according to 
21

$$
\begin{aligned}
& \exp \left\{-\beta\left[f_{1}\left(\operatorname{Tr} M^{\prime} M^{\prime+}\right)^{2}+f_{2} \operatorname{Tr}\left(M^{\prime} M^{\prime+}\right)^{2}\right]\right\}= \\
& =\text { const } \cdot \int_{c-i \infty}^{c+i \infty} \mathcal{D} \Sigma(x) \exp \left\{\operatorname{Tr} \Sigma^{2}+2 \varepsilon \operatorname{Tr}\left(\Sigma M^{\prime} M^{\prime+}\right)+2 \alpha \operatorname{Tr}\left(M^{\prime} M^{\prime}\right) \operatorname{Tr} \Sigma\right\},
\end{aligned}
$$

where $M^{\prime}(x)$ is an $N \times N$-matrix field and

$$
\begin{aligned}
\varepsilon^{2} & =\beta f_{2} \\
2 \varepsilon \alpha+3 \alpha^{2} & =\beta f_{1} .
\end{aligned}
$$

Note that Eqs. (16) are a sophisticated version of the simpler case, where $\phi$ is a scalar field

$$
\text { const } \cdot \exp \left\{-\alpha\left(\phi^{2}(x)\right)^{2}\right\}=\int_{c-i \infty}^{c+i \infty} \mathcal{D} \Sigma(x) e^{\Sigma^{2}(x)-\Sigma(x) \phi^{2}(x) 2 \sqrt{\alpha}} .
$$

Formula (17) can be easily generalized to the case, where the l.h.s. includes also a cubic term $\phi^{3}(x)$, but we are not aware of an analogous transformation that leads to a tractable expression if the cubic term occurs in the form of a determinant. This is the reason why we drop the cubic term in $M_{\ell}^{\prime}$ in the following procedure. The $(3 \times 3)$-matrix field $\Sigma(x)$ is replaced by an $S U(3)$-symmetric diagonal matrix $\Sigma=\operatorname{diag}(\operatorname{sad}, s a d, s a d)$. Thus the matrix of auxiliary fields is reduced to a single field variable $\operatorname{sad}(x)$. The quadratization of the quartic term $\mathcal{L}^{(4)}$ of the Lagrangian leads to

$$
\begin{aligned}
& \mathcal{L}^{(4)}=f_{1}\left(\operatorname{Tr} M^{\prime} M^{\prime+}\right)^{2}+f_{2} \operatorname{Tr}\left(M^{\prime} M^{\prime+}\right)^{2} \\
& \rightarrow \mathcal{L}^{(4) \prime}=\frac{-3}{8\left(3 f_{1}+f_{2}\right)}\left(\frac{1}{2} s a d^{2}+\mu_{0}^{2} s a d\right)+\frac{1}{2}\left(s+\mu_{0}^{2}\right) \operatorname{Tr}\left(M^{\prime} M^{\prime+}\right) .
\end{aligned}
$$

In the saddle-point approximation the path integral $\int \mathcal{D} \Sigma(x)$ is dropped, the auxiliary field $\operatorname{sad}(x)$ is replaced by $s a d^{*}$, which maximizes the integrand. For an $O(N)$-model it is well known that this approximation corresponds to the leading order in a $1 / N$-expansion [22]. In the special case of $f_{2}=0=g$, the $S U(3) \times S U(3)$ sigma-model becomes an $O(18)$-model, which is invariant under $O(18)$-rotations. We have $N=18=2 N_{f}^{2}$, where $N_{f}$ denotes the number of quark flavours, while $N$ labels the number of mesonic modes. Terms of $O(1 / N)$ are dropped, as long as fluctuations in the auxiliary field are neglected. Therefore we call our scheme a large $-N_{f}$ - approximation.

The resulting one-loop contribution to the free energy density is given as

$$
-\frac{1}{\beta V} \ln Z=\frac{1}{2 \beta} \sum_{Q=1}^{8} g(Q) \sum_{n \in Z} \int \frac{d^{3} K}{(2 \pi)^{3}} \ln \left(\beta^{2}\left(\omega_{n}^{2}+\omega_{Q}^{2}\right)\right),
$$


where $g(\pi)=3, g(K)=4, g(\eta)=1, g\left(\eta^{\prime}\right)=1, g\left(\sigma_{\pi}\right)=3, g\left(\sigma_{K}\right)=4, g\left(\sigma_{\eta}\right)=$ $1, g\left(\sigma_{\eta^{\prime}}\right)=1$ are the multiplicity factors of the multiplets, $\omega_{n}^{2} \equiv(2 \pi n / T)^{2}$ and

$$
\begin{aligned}
\omega_{Q}^{2} & \equiv K^{2}+X_{Q}^{2} \\
X_{Q}^{2} & \equiv s a d+\mu_{0}^{2}+m_{Q}^{2} .
\end{aligned}
$$

Thus the one-loop contribution to the free energy takes a form, which is familiar from a free field theory. The only remnant of the interaction is hidden in the effective mass square $X_{Q}^{2}$ via the auxiliary field variable sad. After the sum over the Matsubara frequencies is performed, the full expression for the effective potential is given as

$$
U_{\text {eff }}\left(\sigma_{0}, \sigma_{8}, s a d\right)=U_{\text {class }}\left(\sigma_{0}, \sigma_{8}\right)+U_{\text {saddle }}(s a d)+U_{0}\left(\sigma_{0}, \sigma_{8}, s a d\right)+U_{t h}\left(\sigma_{0}, \sigma_{8}, s a d\right),
$$

where $\sigma_{0}=\bar{\sigma}_{0}, \sigma_{8}=\bar{\sigma}_{8}$ and

$$
\begin{aligned}
& U_{\text {class }}\left(\sigma_{0}, \sigma_{8}\right)=-\frac{1}{2} \mu_{0}^{2}\left(\sigma_{0}^{2}+\sigma_{8}^{2}\right)+\frac{g}{3 \sqrt{3}}\left(2 \sigma_{0}^{3}-\sqrt{2} \sigma_{8}^{3}-3 \sigma_{0} \sigma_{8}^{2}\right) \\
& -\frac{2 \sqrt{2}}{3} f_{2} \sigma_{0} \sigma_{8}^{3}+\left(f_{1}+\frac{f_{2}}{3}\right) \sigma_{0}^{4}+\left(f_{1}+\frac{f_{2}}{2}\right) \sigma_{8}^{4}+2\left(f_{1}+f_{2}\right) \sigma_{0}^{2} \sigma_{8}^{2}-\varepsilon_{0} \sigma_{0}-\varepsilon_{8} \sigma_{8}
\end{aligned}
$$

is the classical part of the potential, which is independent of sad.

$$
U_{\text {saddle }}(\text { sad })=-\frac{3}{8\left(3 f_{1}+f_{2}\right)}\left(\frac{s a d^{2}}{2}+\mu_{0}^{2} s a d\right)
$$

results from the transformation (16), cf. Eq. (18). The one-loop part consists of the zero point energy $U_{0}$ and the thermal part $U_{t h}$, given by

$$
U_{0}\left(\sigma_{0}, \sigma_{8}, s a d\right)=\frac{1}{2} \sum_{Q=1}^{8} g(Q) \int^{\Lambda} \frac{d^{3} K}{(2 \pi)^{3}} \omega_{Q},
$$

$U_{0}$ is divergent if the three-momentum cut-off $\Lambda$ is sent to infinity, while $U_{t h}$ is convergent for $\Lambda \rightarrow \infty$ and vanishes for $T=0$

$$
U_{t h}=\frac{1}{\beta} \sum_{Q=1}^{8} g(Q) \int \frac{d^{3} K}{(2 \pi)^{3}} \ln \left(1-e^{-\beta \omega_{Q}}\right) .
$$

Our results have been obtained for the potential $\hat{U}_{\text {eff }} \equiv U_{\text {eff }}-U_{0}$, where the divergent zero-point energy has just been dropped. It is often argued that the omission of the zero-point energy is justified if one is finally interested in thermodynamic quantities, which are derived as derivatives of $\ln Z$ w.r.t. $\beta$. Usually, $U_{0}$ does not depend on $T$, and the splitting of the one-loop part of $U_{\text {eff }}$ according to $U_{0}$ and $U_{t h}$ is a splitting in a $T$-independent and $T$-dependent part. Thus the contribution of $U_{0}$ should have no effect on the thermodynamics. Strictly speaking we cannot 
use this argument, because $U_{0}$ of Eq. (24) has an implicit temperature dependence hidden in $X_{Q}^{2}$ via $\operatorname{sad}(T)$, the temperature dependent saddle point variable, which is finally chosen such that it maximizes $\hat{U}_{\text {eff }}$ for $s a d=s a d^{*}$. This was one of the reasons why the $U_{0}$-term was kept in [18]. A renormalization prescription was imposed such that the strong (quartic) cut-off dependence of $U_{0}$ was weakened to a $1 / \Lambda^{2}$-dependence. Further differences to [18 are due to corrections of two errors in [18]. After we had removed the programming error in $U_{\text {class }}$ of [18], we found two branches in the free energy density $f$ (or the pressure). The branches in $f$ of the high and low-temperature phases did not cross at some temperature $T=T_{c}$, although the free energy should behave as a smooth function in $T$ by general thermodynamic arguments. The reason was that the two minima of the potential which exist below $T=200[\mathrm{MeV}]$ were erroneously associated to the true minima in the high- and low-temperature phases. It was not realized that for all temperatures up to $T \leq 200[\mathrm{MeV}]$ one minimum is only a local one, while the other one stays the absolute minimum for all temperatures up to $T \geq 200[\mathrm{MeV}]$, cf. also the remarks in section 6 .

Thermodynamic quantities. Thermodynamic observables are derived from the free energy density $f=\lim _{V \rightarrow \infty}\left(-\frac{1}{\beta V} \ln Z\right)$, where $Z$ is approximated as

$$
\hat{Z}\left(\left\langle\sigma_{0}\right\rangle,\left\langle\sigma_{8}\right\rangle\right)=e^{-\beta V \hat{U}_{e f f}\left(\left\langle\sigma_{0}\right\rangle(T),\left\langle\sigma_{8}\right\rangle(T) ; s a d^{*}(T)\right)} .
$$

We have explicitly indicated that $\hat{U}_{\text {eff }}$ should be taken at the saddle point value $\operatorname{sad}^{*}(T)$, which extremizes $\hat{U}_{\text {eff }}$. The expression for the energy density $\varepsilon$, entropy density $s$, and the pressure $p$ are derived in the standard way. We have

$$
\begin{aligned}
p & =-\hat{U}_{e f f}, \\
s & =-\left(\frac{\partial f}{\partial T}\right)=(p+\varepsilon) / T \\
\varepsilon & =-\frac{\partial \ln \hat{Z}}{\partial \beta}=\sum_{Q=1}^{8} g(Q) \frac{T^{4}}{2 \pi^{2}} \int_{0}^{\infty} d \alpha\left(\frac{\alpha^{2} \sqrt{\alpha^{2}+y_{Q}^{2}}}{e^{\sqrt{\alpha^{2}+y_{Q}^{2}}}-1}\right) \\
\alpha & \equiv K / T, y_{Q} \equiv\left(\sqrt{s a d^{*}+\mu_{0}^{2}+m_{Q}^{2}\left(\left\langle\sigma_{0}\right\rangle,\left\langle\sigma_{8}\right\rangle\right)}\right) / T .
\end{aligned}
$$

Evaluation of $\hat{U}_{\text {eff }}$. Next let us discuss the evaluation of $\hat{U}_{\text {eff }}$ in some more detail. The expression for $\hat{U}_{\text {eff }}$ is familiar from the expression for a free field theory. In a high-temperature expansion it reads

$$
\hat{U}_{\text {eff }}=U_{\text {class }}+U_{\text {saddle }}-\frac{T^{4}}{2 \pi^{2}} \sum_{Q=1}^{8} g(Q)\left\{\frac{\pi^{4}}{45}-\frac{\pi^{2}}{12} y_{Q}^{2}+O\left(y_{Q}^{3}\right)\right\}
$$


with $y_{Q}=\frac{X_{Q}}{T}$.

At high temperature the $S U(3) \times S U(3)$ linear sigma-model certainly fails to describe the quark-gluon plasma phase. The critical temperature falls neither in a high nor in a low-temperature region, the expansion parameter is of $O(1)$ or larger. Nevertheless, we discuss the high-temperature expansion, which has been frequently applied in calculations of a thermodynamic potential. It has the advantage that imaginary parts of $\hat{U}_{e f f}$ are absent to leading order in $X_{Q} / T$, cf. e.g. 23. We have performed a high-temperature expansion for two sets of mass parameters, the chiral limit and the realistic mass point, see sections 4 and 7 . This way we got some qualitative insight in the phase structure before we could tackle the problems in a fully numerical evaluation of $\hat{U}_{\text {eff }}$. Analytic expressions for a low-temperature expansion of $\hat{U}_{\text {eff }}$ are known as well [24. We have used them only in intermediate steps to check the numerics. Thus we turn now to the fully numerical evaluation of $\hat{U}_{\text {eff }}$.

At a first glance the numerical evaluation of $\hat{U}_{\text {eff }}$ looks rather straightforward. For each pair of $\left(\sigma_{0}, \sigma_{8}\right)$ we have to find $s a d^{*}$ that maximizes $\hat{U}_{e f f}$. The condensates $\left\langle\sigma_{0}\right\rangle,\left\langle\sigma_{8}\right\rangle$ are then determined as minima of $\hat{U}_{\text {eff }}\left(\sigma_{0}, \sigma_{8} ; \operatorname{sad}^{*}\left(\sigma_{0}, \sigma_{8}\right)\right)$. It is well known 23] that the arguments $\left(K^{2}+X_{Q}^{2}\right)$ of the logarithm in $\hat{U}_{\text {eff }}$ can become negative and lead to imaginary parts in $\hat{U}_{\text {eff }}$, which we have mentioned above. The original hope was that the positive contribution of the auxiliary field sad to the masses $m_{Q}$ helps in avoiding imaginary parts of $\hat{U}_{\text {eff }}$. Actually no imaginary parts have been found in [21]. As can be seen from Fig. 2 the contribution of $s^{*}$ to $X_{Q}^{2}$ increases with $T$, it is positive for $T \geq 116[\mathrm{MeV}]$, but in our case the positive contribution is not sufficient to avoid negative $X_{Q}^{2}$ completely, i.e. for all $\sigma_{0}, \sigma_{8}$ and $T$. It has turned out that the actual minima $\left\langle\sigma_{0}\right\rangle,\left\langle\sigma_{8}\right\rangle$ lie always in the "allowed" region of real valued $\hat{U}_{\text {eff }}$ or at least at the boundary of this region, but on the way of searching the maximum in $s a d^{*}$ and the minima in $\left\langle\sigma_{0}\right\rangle,\left\langle\sigma_{8}\right\rangle$ the routines encounter negative mass squares indispensably. Therefore $\hat{U}_{\text {eff }}$ is analytically continued in the following way. The integral in $U_{t h}$ of $\hat{U}_{\text {eff }}$ is of the type

$$
I(z)=\int_{0}^{\infty} d x\left\{x^{2} \ln \left[1-\exp \left[-\left(x^{2}+z^{2}\right)^{1 / 2}\right]\right\}=-z^{2} \sum_{n=1}^{\infty} \frac{1}{n^{2}} K_{2}(n z)\right.
$$

where $K_{2}$ is a modified Bessel function. The analytic continuation of $K_{m}(n z)$ to complex values of $n z$ is given as

$$
K_{m}(n z)=i^{m+1}\left(\frac{\pi}{2}\right)\left[J_{m}(i n z)+i Y_{m}(i n z)\right]
$$

where $J_{m}$ are Bessel functions of the first kind and $Y_{m}$ are Weber functions 25. 
Figure 2: Saddle point $s a d^{*}$ as a function of temperature $T$

This leads to the following form of $\hat{U}_{\text {eff }}$

$$
\hat{U}_{\text {eff }}=U_{\text {class }}+U_{\text {saddle }}+\frac{T^{4}}{2 \pi^{2}} \sum_{Q=1}^{8} g(Q)\left\{\frac{-X_{Q}^{2}}{T^{2}} \sum_{n=1}^{\infty} \frac{1}{n^{2}} K_{2}\left(\frac{n}{T} X_{Q}\right)\right\}
$$

for $X_{Q}^{2} \geq 0$. For $X_{Q}^{2}<0$ and $X_{Q}= \pm i \sqrt{\left|X_{Q}^{2}\right|}$

$$
\hat{U}_{\text {eff }}=U_{\text {class }}+U_{\text {saddle }}+\operatorname{Re} U_{t h}+i \operatorname{Im} U_{t h}
$$

with

$$
\begin{aligned}
& \operatorname{Re} U_{t h}=\frac{T^{4}}{2 \pi^{2}} \sum_{Q=1}^{8} g(Q)\left\{\frac{\pi}{2} \frac{\left|X_{Q}^{2}\right|}{T^{2}} \sum_{n=1}^{\infty} \frac{1}{n^{2}} Y_{2}\left(\frac{n}{T}\left|X_{Q}^{2}\right|^{1 / 2}\right)\right\} \\
& \operatorname{Im} U_{t h}=\frac{T^{4}}{2 \pi^{2}} \sum_{Q=1}^{\infty} g(Q)\left\{ \pm \frac{\pi}{2} \frac{\left|X_{Q}^{2}\right|}{T^{2}} \sum_{n=1}^{\infty} \frac{1}{n^{2}} J_{2}\left(\frac{n}{T}\left|X_{Q}^{2}\right|^{1 / 2}\right)\right\} .
\end{aligned}
$$

In the actual calculation we have cut the infinite sums in Eq. (33),(34) after 25 terms. 
The condensate values $\left\langle\sigma_{0}\right\rangle(T),\left\langle\sigma_{8}\right\rangle(T)$ are determined as minima of $\hat{U}_{\text {eff }}$ for $X_{Q}^{2} \geq 0$ and of Re $\hat{U}_{\text {eff }}$ for $X_{Q}^{2}<0$.

Error analysis. There are mainly two sources for errors. The first one is the determination of the saddle point from the condition $\partial \hat{U}_{\text {eff }} / \partial s a d=0$. In searching $s a d^{*}$ the routines encounter negative values of $X_{Q}^{2}$ in intermediate steps and find $s a d^{*}$ from derivatives of Weber functions $Y_{2}\left(\frac{n}{T}\left|X_{Q}^{2}\right|^{1 / 2}\right)$, see Eq. (34). The Weber functions are taken from representations in [26] which cover the range from small to large arguments. For arguments $\frac{n}{T}\left|X_{Q}^{2}\right|^{1 / 2} \geq 4.8(9.7), Y_{2}\left(\frac{n}{T}\left|X_{Q}^{2}\right|^{1 / 2}\right) \leq 6.0 \cdot 10^{-3}\left(2.9 \cdot 10^{-5}\right)$. Due to the large variation of the effective masses the arguments become easily $\geq 100$. Thus for small temperatures, large effective masses and/or large values of $n$ the contributions to the sum over $n$ are so small that they reach the order of the numerical accuracy. The location of the saddle point becomes inaccurate under these conditions. Similarly, for small arguments (i.e. high temperatures, small values of $X_{Q}$ ) the approximations of the Weber functions in the vicinity of their singularity at vanishing arguments become less reliable [26]. This explains why the error in $s a d^{*}$ increases with temperature, if simultaneously $\left|X_{Q}^{2}\right|^{1 / 2}$ becomes smaller.

The error $\Delta s a d^{*}$ in the maximum $s a d^{*}$ of $\hat{U}_{\text {eff }}$ is estimated as the difference between the zeroes of $\partial \hat{U}_{e f f} / \partial s a d=0$ if $s a d^{*}$ is extrapolated from the right or approached from the left in the following way. On the left hand side of the maximum, $\operatorname{Re} \hat{U}_{\text {eff }}$ is represented by the series of Weber functions, it is oscillating and $\partial \hat{U}_{\text {eff }} / \partial s a d>0$. The zero of the oscillating derivative is actually used in the program as value for $s a d^{*}$. The oscillations extend to some region (of temperature dependent size) on the right hand side of $\partial \hat{U}_{\text {eff }} / \partial s a d=0$, where the derivative is negative. For further increasing values of sad the effective potential becomes real and behaves approximately quadratically in sad, such that $\partial \hat{U}_{e f f} / \partial s a d$ is linear in sad. We extrapolate the linear part of the derivative to zero and use the extrapolated zero as second value for $s a d^{*}$. The difference between both values for $s a d^{*}$ gives an estimate for the ambiguity in finding the maximum of $\hat{U}_{\text {eff }}$ from the parabolic or the oscillating behaviour. It turns out that for low temperatures $\Delta s a d^{*} \sim 0$, while it increases with temperature to $\Delta s a d^{*} \sim \pm 0.006[\mathrm{GeV}]^{2}$ in the transition region in case of the chiral limit, cf. Fig. 2.

The uncertainty in the determination in $s^{*} d^{*}$ leads to errors in the meson condensates, the effective masses $X_{Q}$, and the thermodynamic quantities $\varepsilon, p$, and $s$. The errors $\Delta\left\langle\sigma_{0,8}\right\rangle$ which are induced in $\left\langle\sigma_{0}\right\rangle,\left\langle\sigma_{8}\right\rangle$ due to the uncertainty in the precise value of $s a d^{*}$ are estimated from

$$
\Delta\left\langle\sigma_{0,8}\right\rangle=\left.\frac{-\partial^{2} \hat{U}_{e f f} / \partial s a d^{*} \partial \sigma_{0}}{\partial^{2} U_{e f f} / \partial^{2} \sigma_{0}^{2}}\right|_{\left\langle\sigma_{0}\right\rangle,\left\langle\sigma_{8}\right\rangle, s a d^{*}} \cdot \Delta s a d^{*}
$$


since $\left\langle\sigma_{0,8}\right\rangle\left(\operatorname{sad}^{*}\right)$ are implicitly determined via $\partial \hat{U}_{e f f} / \partial \sigma_{0,8}=0$. In case of realistic masses this leads to $\Delta\left\langle\sigma_{0,8}\right\rangle \leq 10^{-7}$ below $T \sim 50 \mathrm{MeV}, \leq 10^{-4}$ below $T \sim 125 \mathrm{MeV}$, $\leq 10^{-3}$ for $T \leq 165 \mathrm{MeV}$ and of the order of $10^{-3}[\mathrm{MeV}]$ in the transition region. After the crossover $(T \geq 197 \mathrm{MeV})$ it stays $\sim O\left(10^{-4}\right)$.

An independent second source for errors in the condensates $\left\langle\sigma_{0}\right\rangle,\left\langle\sigma_{8}\right\rangle$ is the flat shape of the effective potential in the transition region, in particular for external field strengths which only admit a rather weak first order phase transition. We estimate $\Delta\left\langle\sigma_{0}\right\rangle\left(\simeq \Delta\left\langle\sigma_{8}\right\rangle\right)$ from plots of $\hat{U}_{\text {eff }}\left(\sigma_{0}\right)$ in the $S U(3)$-symmetric case. We find $\Delta\left\langle\sigma_{0}\right\rangle \simeq \pm 1.3[\mathrm{MeV}]$ in the chiral limit and $\simeq \pm 4.3[\mathrm{MeV}]$ for $\epsilon_{0}=2 \cdot 10^{-4}$ or $2.5 \cdot 10^{-4}[\mathrm{GeV}]^{3}$, cf. Fig. 6 of section 5 .

In the $S U(3)$-symmetric case $\left\langle\sigma_{8}\right\rangle$ should identically vanish for all temperatures. The eight Goldstone bosons are identical in mass. If the $S U(3)$-symmetric case is calculated as a special case $\left(\varepsilon_{0} \neq 0=\varepsilon_{8}\right)$ of our general framework $\left(\varepsilon_{0} \neq 0 \neq\right.$ $\left.\varepsilon_{8}\right),\left\langle\sigma_{8}\right\rangle=0$ should come out automatically. In fact it vanishes within the numerical accuracy for temperatures $T \leq 50[\mathrm{MeV}]$ for $\epsilon_{0}=2.5 \cdot 10^{-4}[\mathrm{GeV}]^{3}$. In the transition region $\left\langle\sigma_{8}\right\rangle$ strongly fluctuates around zero. The maximal fluctuation varies between 0.2 and $3.0[\mathrm{MeV}]$ depending on $\epsilon_{0}$ and the strength of the first order transition. The values are of the same order of magnitude as $\Delta\left\langle\sigma_{0,8}\right\rangle$ due to the flat shape of the effective potential.

When the meson condensates are converted to the quark condensates, additional errors enter due to the current quark masses. We use $\Delta m_{u, d}= \pm 1.45[\mathrm{MeV}]$ [19].

Finally, the errors in the entropy and energy densities $s$ and $\varepsilon$ can be traced back to the effective masses $X_{Q}\left(\left\langle\sigma_{0}\right\rangle,\left\langle\sigma_{8}\right\rangle, s a d^{*}\right)$. The resulting errors in $s / T^{3}$ in the crossover region are indicated in Fig. 10 of section 6 , where $s / T^{3}$ is shown for realistic meson masses.

From very different approximation schemes it is known that the region of a phase transition is particularly difficult to handle. We consider our difficulties in localizing the saddle point and calculating the condensates in the transition/crossover region just as a special manifestation of that.

\section{The chiral limit}

From the renormalization group analysis of Pisarski and Wilczek [1] we expect a first order chiral transition in the chiral limit. As mass input we choose $m_{\pi}^{2}=m_{K}^{2}=$ $m_{\eta}^{2}=0$ for the pseudoscalar Goldstone bosons, $m_{\eta^{\prime}}=850[\mathrm{MeV}], m_{\sigma_{\eta^{\prime}}}=800[\mathrm{MeV}]$, 
$f_{\pi}=94[\mathrm{MeV}]$. The mass of the sigma meson should be treated as a parameter due to the uncertainty in the experimental identification. For the final parametrization we choose $m_{\sigma_{\eta}}=600[\mathrm{MeV}]$.

The tree level parametrization of the Lagrangian (1) in the limit of vanishing $\varepsilon_{0}$ and $\varepsilon_{8}$ is then given by

$$
\begin{aligned}
\mu_{0}^{2} & =5.96 \cdot 10^{-2}[\mathrm{GeV}]^{2} \\
f_{1} & =4.17 \\
f_{2} & =4.48 \\
g & =-1.81[\mathrm{GeV}] .
\end{aligned}
$$

The high-temperature expansion gives a 1 st order transition at $T_{c}=92 \pm 1[\mathrm{MeV}]$,

Figure 3: Normalized light quark condensate $\langle\bar{q} q\rangle_{T} /\langle\bar{q} q\rangle_{0}$ as a function of temperature $T$ in the high-temperature expansion (solid curve) and the numerical evaluation (dashed curve) of the large $N_{f}$-expansion. The condensate drops to zero at $T_{c} \sim 92[\mathrm{MeV}]$ and $177[\mathrm{MeV}]$, respectively.

while the fully numerical evaluation leads to $T_{c}=177 \pm 1[\mathrm{MeV}]$, cf. Fig. 3 . This is in 
agreement with the general observation that the high- $T$-expansion gives qualitatively correct results when it is extrapolated beyond its validity range, quantitatively it fails in precise predictions. The $T_{c}$-value of the numerical calculation supports the estimate for the $N_{f}$-dependence of $T_{c}$, which has been derived by Cleymans, Kocić and Scadron [27] from a pion gas model without interactions as

$$
T_{c} \sim 2 \cdot f_{\pi} \cdot \sqrt{3 N_{f} /\left(N_{f}^{2}-1\right)} \sim 200[\mathrm{MeV}]
$$

The effective potential $\hat{U}_{\text {eff }}$ is plagued with imaginary parts for all temperatures we have investigated, i.e. between 0 and $250[\mathrm{MeV}]$. When we evaluate $\operatorname{Re} \hat{U}_{\text {eff }}$ according to Eq. (34), we observe the same effect for higher values of the sigmamass as it has been noticed by Goldberg [20]. Goldberg has calculated the effective potential of the $S U(3) \times S U(3)$ sigma-model in a mean-field approximation in the chiral limit. The oscillations in the potential became stronger for larger values of the $\sigma$-meson mass, which was used as input. In our case Re $\hat{U}_{e f f}\left(\sigma_{0}\right)$ strongly fluctuates around the expected parabola if $m_{\sigma} \geq 1[\mathrm{GeV}]$. This explains our final parameter choice of $m_{\sigma_{\eta}}=600[\mathrm{MeV}]$. The approximation of Re $\hat{U}_{\text {eff }}$ according to Eq. (34) by a finite series of Weber functions $(n \leq 25)$ loses its validity if the argument of $Y_{2}$ becomes too large due to $m_{\sigma}^{2}$.

For the barrier height between the coexisting minima of the potential we find 0.14 . $10^{-3}\left[\mathrm{GeV} / \mathrm{fm}^{3}\right]$. The barrier height may be regarded as a measure for the strength of the transition. It determines the tunneling rate between coexisting phases in the transition region. The barrier height is clearly smaller than the value of Frei and Patkós [21], who found $0.36\left[\mathrm{GeV} / \mathrm{fm}^{3}\right]$ in the same model, but without inclusion of the $n \neq 0$-Matsubara frequencies. The higher barrier goes along with a large value for the interface tension $\alpha$ between the coexisting chirally broken and symmetric phases at $T_{c}, \alpha$ has been estimated as $[(40-50) \mathrm{MeV}]^{3}[21]$. More recent lattice results indicate that such a large value is not likely for QCD 28.

Of particular interest in the chiral limit is the temperature dependence of the effective masses $X_{Q}^{2}=s a d+\mu_{0}^{2}+m_{Q}^{2}, Q=1, \ldots, 8$. In the chiral limit the masses are free of contributions of the external fields. Thus the Goldstone theorem should be obeyed by the pseudoscalar meson octet. A short glance at Fig. 4 shows that the effective masses $X_{Q}=X_{\pi}=X_{K}=X_{\eta}$ do not stay massless for $T>0$. They get an increasingly positive contribution with increasing $T$ due to the saddle point contribution $\operatorname{sad}^{*}(T)$. If the physical meaning of $X_{Q}$ coincides to leading order in $1 / N_{f}$ with a dynamical mass (in particular if the dynamical and screening masses coincide to leading order in $1 / N_{f}$ and both are equal to $\left.X_{Q}\right)$, the increase of $X_{Q}(T)$ with $T$ would violate Goldstone's theorem and should be considered as an artifact 
Figure 4: Effective masses $X_{Q}, Q=1, \ldots, 8$ as a function of $T$ in the chiral limit. In units of $[\mathrm{MeV}]$ we have at $T=0 X_{\pi}=X_{K}=X_{\eta}=0, X_{\eta^{\prime}}=850, X_{\sigma_{\eta^{\prime}}}=600$, $X_{\sigma_{\pi}}=X_{\sigma_{K}}=X_{\sigma_{\eta}}=800$. The masses are degenerate at $T_{c} \sim 177 \pm 3[\mathrm{MeV}]^{\eta^{\prime}}$.

of our approximation. We still have to clarify this point.

\section{The critical transition line}

The critical transition line in an $\left(m_{\pi}, m_{K}, \ldots\right)$-diagram consists of pseudoscalar meson masses for which the first order chiral transition becomes of second order and turns into a crossover phenomenon for meson masses exceeding the critical values. We will determine three such critical points. The critical points are characterized by their external field strengths $\varepsilon_{0}, \varepsilon_{8}$. The first point corresponds to an $S U(3)$ symmetric case, where $\varepsilon_{8}=0, m_{u}=m_{d}=m_{s} \neq 0$ and $m_{\pi}=m_{K}=m_{\eta} \neq 0$. Since $\left\langle\sigma_{8}\right\rangle=0$ for all temperatures, the numerics considerably simplifies compared to the general case with $\varepsilon_{0} \neq 0 \neq \varepsilon_{8}$. For the second critical point we choose 
$\varepsilon_{0}=-0.77 \varepsilon_{8}$. This ratio is identical to $\varepsilon_{0} / \varepsilon_{8}$ for the mass point with realistic meson masses, where $m_{s} / \hat{m}=18.2$, cf. section 2 . The third point is characterized by $\varepsilon_{0}=(2 \alpha / \beta) \cdot \varepsilon_{8}$. It is chosen such that $m_{s}=0, m_{u, d} \neq 0$. Before we present our results for the critical field strengths in the large- $N_{f}$-expansion, we calculate $\varepsilon_{0}^{\text {crit }}, \varepsilon_{8}^{\text {crit }}$ in a mean-field approximation.

\subsection{Critical meson masses in a mean-field approximation}

Recently, Gavin, Goksch and Pisarski [17] have calculated a set of critical quark masses in the linear $S U(3) \times S U(3)$ sigma-model. The calculation has been performed in a mean-field approximation. Since we use a different tree-level parametrization of the sigma-model, we have reproduced the mean-field calculation for our parameter choice and summarize the main steps.

The $S U(3)$-symmetric case. Let us first consider the $S U(3)$-symmetric case with $\varepsilon_{8}=0$. In mean-field we have to deal with the classical part of the potential $U_{\text {class }}$, which follows from the Lagrangian (1) for a constant background field $\sigma_{0}=\bar{\sigma}_{0}\left(\bar{\sigma}_{8}=\right.$ $0)$. We have

$$
U_{\text {class }}\left(\sigma_{0}\right)=-\frac{1}{2} \mu_{0}^{2} \sigma_{0}^{2}+\frac{2}{3 \sqrt{3}} g \sigma_{0}^{3}+\left(f_{1}+\frac{f_{2}}{3}\right) \sigma_{0}^{4}-\varepsilon_{0} \sigma_{0} .
$$

This form is familiar from Landau's free energy functional in terms of an order parameter field. One of its applications is a description of the phase structure for a liquid/gas transition. For $\varepsilon_{0}=0$ the system has a first order transition from the liquid to the gas phase. The transition stays first order, until the external field (the pressure in case of a liquid/gas system) is increased to a critical value $\varepsilon_{0}^{c r i t}$, where it becomes second order. For values of $\varepsilon_{0}>\varepsilon_{0}^{\text {crit }}$, the transition is washed out and turns into a crossover phenomenon.

In mean-field the effect of a finite (and strictly speaking high) temperature is reduced to a renormalization of the mass parameter term, i.e. of the coefficient of the quadratic term in the Lagrangian. Thus the finite temperature effects can be mimiced by tuning $\mu_{0}^{2}$ while keeping the other couplings in the Lagrangian $\left(f_{1}, f_{2}, g\right)$ fixed. The potential can be Taylor expanded around its true minimum $\sigma_{0}^{\text {min }}$ (which is different from zero in the symmetry broken phase); in particular it can be expanded around the "critical" $\sigma_{0}^{\text {min }} \equiv \sigma_{0}^{\text {crit }}$ for the critical field strength $\varepsilon_{0}^{\text {crit }}$. In the "critical" case $U_{\text {class }}$ starts with a term quartic in $\left(\sigma_{0}-\sigma_{0}^{\text {crit }}\right)$. From the vanishing of the first three coefficients we obtain the critical parameters as follows.

Since the first order transition just disappears for $\varepsilon_{0}^{\text {crit }}$, the third derivative of $U_{\text {class }}$ 
w.r.t. $\sigma_{0}$ should vanish at $\sigma_{0}=\sigma_{0}^{\text {crit }}$ leading to

$$
\sigma_{0}^{\text {crit }}=\frac{-g}{\sqrt{3} \cdot 6\left(f_{1}+f_{2} / 3\right)}=3.1 \cdot 10^{-2}[\mathrm{GeV}]
$$

The second derivative $\partial^{2} U_{\text {class }} / \partial \sigma_{0}^{2}$ at $\sigma_{0}^{\text {crit }}$ is the coefficient of the quadratic fluctuations $\left(\sigma_{0}-\sigma_{0}^{c r i t}\right)^{2}$ around $\sigma_{0}^{c r i t}$, thus it has the meaning of $m_{\sigma_{\eta^{\prime}}}^{2}$. This is the critical mass, which goes to zero when the second order transition is approached. It is easily checked that the other meson masses remain finite for the same set of critical parameters. The vanishing of $m_{\sigma_{\eta^{\prime}}}^{2}$ at criticality or $\partial^{2} U_{\text {class }} / \partial \sigma_{0}^{2}$ at $\sigma_{0}^{\text {crit }}$ implies for the critical value of $\mu_{0}^{2}$

$$
\mu_{0}^{2}{ }^{c r i t}=\frac{-g^{2}}{9 \cdot\left(f_{1}+f_{2} / 3\right)}=-6.44 \cdot 10^{-2}[\mathrm{GeV}]^{2} .
$$

Finally the extremum condition for $\sigma_{0}^{\text {crit }}$ determines $\varepsilon_{0}^{\text {crit }}$ as

$$
\varepsilon_{0}^{c r i t}=-\frac{1}{27 \cdot 6 \cdot \sqrt{3}} \frac{g^{3}}{\left(f_{1}+f_{2} / 3\right)^{2}}=6.6 \cdot 10^{-4}[\mathrm{GeV}]^{3} \text {. }
$$

The value for $\varepsilon_{0}^{\text {crit }}=6.6 \cdot 10^{-4}[\mathrm{GeV}]^{3}\left(\varepsilon_{8}^{\text {crit }}=0\right)$ leads to a pseudoscalar octet mass of $m_{\pi}=m_{K}=m_{\eta}=146[\mathrm{MeV}]$ and to a current quark mass of $m_{u}=$ $m_{d}=m_{s}=1.9[\mathrm{MeV}]$. One should keep in mind that the tree level parametrization of the $S U(3) \times S U(3)$ sigma-model is arbitrary to some extent. If we would choose $g=-1.39[\mathrm{GeV}], f_{1}=5.3, f_{2}=0.93$ with $m_{\sigma_{\eta^{\prime}}}=600[\mathrm{MeV}]$ (the values which have been used in [11] for the tree level parametrization), $\varepsilon_{0}^{\text {crit }}$ turns out as $3 \cdot 10^{-4}[\mathrm{GeV}]^{3}$ or $m_{u, d, s} \simeq 0.9[\mathrm{MeV}]$ leading to $\left\langle m_{p s}\right\rangle=115[\mathrm{MeV}]$. For the same parameter choice, but $f_{1}=2.35$ and $m_{\sigma_{\eta}}=950[\mathrm{MeV}], \varepsilon_{0}^{\text {crit }}$ comes out as $1.3 \cdot 10^{-3}[\mathrm{GeV}]^{3}$. The same tendency has been observed in [17]. An increase of the input mass $m_{\sigma_{\eta}}$ shifts the critical field strength to larger values (reducing the discrepancy to the lattice result). The parameter choice which has been used in [18] leads to $\varepsilon_{0}^{\text {crit }}=4 \cdot 10^{-4}[\mathrm{GeV}]^{3}$. Gavin, Goksch and Pisarski [17] obtain for the corresponding critical field strength in the $S U(3)$-symmetric case $h_{0}^{\text {crit }}=1.6 \cdot 10^{-4}[\mathrm{GeV}]^{3}$. Due to their different parametrization it is not identical with ours, but of the same order of magnitude. Thus we estimate the error in the mean-field calculation as $\Delta \epsilon_{0}^{\text {crit }}= \pm 5 \cdot 10^{-4}[\mathrm{GeV}]^{3}$. The induced error in the pseudoscalar meson masses comes out as large as $\approx 127[\mathrm{MeV}]$, in the current light quark mass it is $\leq 1.4[\mathrm{MeV}]$.

The general case of $\varepsilon_{0} \neq 0, \varepsilon_{8} \neq 0$. For non-vanishing $\varepsilon_{0}$ and $\varepsilon_{8} U_{\text {class }}$ is given by Eq. (22). Again we have to determine the critical parameter $\mu_{0}^{2}$ to simulate a 
finite temperature, the critical minima values $\sigma_{0}^{\text {crit }}, \sigma_{8}^{\text {crit }}$ of $U_{\text {class }}$ and the critical field strengths $\varepsilon_{0}^{c r i t}, \varepsilon_{8}^{c r i t}$. We use the following conditions

$$
\begin{aligned}
\partial U_{\text {class }}\left(\sigma_{0}, \sigma_{8}\right) /\left.\partial \sigma_{0}\right|_{\text {crit }} & =0 \\
\partial U_{\text {class }}\left(\sigma_{0}, \sigma_{8}\right) /\left.\partial \sigma_{8}\right|_{\text {crit }} & =0 \\
\left.m_{\sigma_{\eta^{\prime}}}^{2}\right|_{\text {crit }} & =0 \\
\varepsilon_{0}^{\text {crit }} / \varepsilon_{8}^{\text {crit }}+\left.0.77\right|_{\text {crit }} & =0 \\
\partial^{3} U_{\text {class }}\left(\sigma_{0}, \sigma_{8}\right) /\left.\partial r^{3}\right|_{\text {crit }} & =0 .
\end{aligned}
$$

The five conditions are postulated at criticality (abbreviated as $\left.\right|_{\text {crit }}$ ), i.e. for the set of critical parameters $\sigma_{0}^{\text {crit }}, \sigma_{8}^{\text {crit }}, \varepsilon_{0}^{\text {crit }}, \varepsilon_{8}^{\text {crit }}, \mu_{0}^{2}$ crit. The first three equations are obvious generalizations of the $S U(3)$-symmetric case. Eq. (45) is just one possible choice saying that we keep the mass splitting of realistic (pseudo)scalar meson masses fixed in the tuning to the critical transition line. Eq. (46) generalizes $\partial^{3} U_{\text {class }}\left(\sigma_{0}\right) /\left.\partial \sigma_{0}^{3}\right|_{\text {crit }}=0$ of the one-dimensional case. More precisely, it postulates that the directional derivative in the radial direction $r$ in $\left(\sigma_{0}, \sigma_{8}\right)$-space (i.e. perpendicular to the direction of $m_{\sigma_{\eta^{\prime}}}^{2}=0$ ) should vanish to exclude the occurrence of a first order transition. Eqs. (42-43) lead to

$$
\begin{aligned}
\varepsilon_{0}^{c r i t}= & -\mu_{0}^{2} \sigma_{0}+\frac{g}{\sqrt{3}}\left(2 \sigma_{0}-\sigma_{8}\right)-\frac{2}{3} \sqrt{2} f_{2} \sigma_{8}^{3}+4\left(f_{1}+f_{2} / 3\right) \sigma_{0}+4\left(f_{1}+f_{2}\right) \sigma_{0} \sigma_{8}^{2} \\
\varepsilon_{8}^{c r i t}= & -\mu_{0}^{2} \sigma_{8}-\sqrt{\frac{2}{3}} \cdot g\left(\sigma_{8}^{2}+\sqrt{2} \sigma_{0} \sigma_{8}\right)-2 \sqrt{2} f_{2} \sigma_{0} \sigma_{8}^{2}+ \\
& +4\left(f_{1}+f_{2} / 2\right) \sigma_{8}^{3}+4\left(f_{1}+f_{2}\right) \sigma_{0}^{2} \sigma_{8},
\end{aligned}
$$

where finally $\mu_{0}^{2}=\mu_{0}^{2}$ crit $, \sigma_{0}=\sigma_{0}^{\text {crit }}, \sigma_{8}=\sigma_{8}^{\text {crit }}$.

If we introduce the auxiliary quantities $r_{0}, r_{8}$ according to

$$
\begin{aligned}
& r_{0} \equiv\left(12 f_{1}+4 f_{2}\right) \sigma_{0}^{2}+4\left(f_{1}+f_{2}\right) \sigma_{8}^{2}+4 g \sigma_{0} / \sqrt{3} \\
& r_{8} \equiv 4\left(f_{1}+f_{2}\right) \sigma_{0}^{2}+6\left(2 f_{1}+f_{2}\right) \sigma_{8}^{2}-4 \sqrt{2} f_{2} \sigma_{0} \sigma_{8}-\frac{2 g}{\sqrt{3}} \sigma_{0}-2 \sqrt{\frac{2}{3}} g \sigma_{8}
\end{aligned}
$$

and use $m_{\sigma_{08}}^{2}$ of Eq. (11), Eq. (44) implies

$$
\mu_{0}^{2}{ }^{c r i t}=\frac{1}{2}\left[r_{0}+r_{8}-\sqrt{\left(r_{0}-r_{8}\right)^{2}+4\left(m_{\sigma_{08}}^{2}\right)^{2}}\right],
$$

where in the end $\sigma_{0}=\sigma_{0}^{\text {crit }}, \sigma_{8}=\sigma_{8}^{\text {crit }}$ in Eqs. (48).

So far we have $\varepsilon_{0,8}^{\text {crit }}=\varepsilon_{0,8}^{\text {crit }}\left(f_{1}, f_{2}, g, \sigma_{0}, \sigma_{8}\right)$ and $\mu_{0}^{2}{ }^{\text {crit }}=\mu_{0}^{2}{ }^{\text {crit }}\left(f_{1}, f_{2}, g, \sigma_{0}, \sigma_{8}\right)$. Finally the zeroes of Eqs. (45-46) determine $\sigma_{0}^{\text {crit }}, \sigma_{8}^{\text {crit }}$. These equations are solved numerically. We find

$$
\sigma_{0}^{\text {crit }}=3.6 \cdot 10^{-2}[\mathrm{GeV}], \sigma_{8}^{\text {crit }}=-5.5 \cdot 10^{-3}[\mathrm{GeV}]
$$


Using these values, Eqs. (49), (47) and (48) give

$$
\begin{aligned}
\mu_{0}^{2}{ }^{\text {crit }} & =-6.5 \cdot 10^{-2}[\mathrm{GeV}]^{2}, \\
\varepsilon_{0}^{\text {crit }} & =7.7 \cdot 10^{-4}[\mathrm{GeV}]^{3}, \quad \varepsilon_{8}^{\text {crit }}=-1.0 \cdot 10^{-3}[\mathrm{GeV}]^{3}
\end{aligned}
$$

or $m_{s}=6[\mathrm{MeV}], \hat{m}=0.3[\mathrm{MeV}]$, while the average pseudoscalar meson mass $\left\langle m_{p s}\right\rangle$ $=126.4[\mathrm{MeV}]$. The values for $\varepsilon_{0,8}^{\text {crit }}$ are compatible with the results of Gavin, Goksch and Pisarski [17], who find for the critical field strengths $h_{0}^{\text {crit }}=(62[\mathrm{MeV}])^{3}, h_{8}^{\text {crit }}=$ $(60.4[\mathrm{MeV}])^{3}$, if one keeps in mind the different tree level parametrization. For example $m_{s} / m_{u, d}=32$ in [17], while $m_{s} / m_{u, d}=18.2$ in our case.

The case of $m_{s}=0$ or $\varepsilon_{0} / \varepsilon_{8}=2 \alpha / \beta$. If we replace 0.77 in Eq. (45) by $-2 \alpha / \beta=$ -1.31 corresponding to $m_{s}=0, m_{u, d} \neq 0$, we obtain from Eqs. (42)-(46)

$$
\begin{array}{rlrl}
\sigma_{0}^{\text {crit }} & =3.4 \cdot 10^{-2}[\mathrm{GeV}], \quad \sigma_{8}^{\text {crit }}=2.9 \cdot 10^{-3}[\mathrm{GeV}] \\
\mu_{0}^{2}{ }^{\text {crit }} & =-6.4 \cdot 10^{-2}[\mathrm{GeV}]^{2} & \\
\varepsilon_{0}^{\text {crit }} & =6.7 \cdot 10^{-4}[\mathrm{GeV}]^{3}, \quad \varepsilon_{8}^{\text {crit }}=5.15 \cdot 10^{-4}[\mathrm{GeV}]^{3}
\end{array}
$$

or $\hat{m}=2.9[\mathrm{MeV}]$ and $\left\langle m_{p s}\right\rangle=137.2[\mathrm{MeV}]$. Note that the condition $m_{s}=0$ implies the same sign for $\varepsilon_{0}^{\text {crit }}$ and $\varepsilon_{8}^{\text {crit }}$ due to the same sign for the constants $\alpha$ and $\beta$. Hence the "critical" condensates $\sigma_{0,8}^{\text {crit }}$ come out with equal sign in contrast to the realistic mass case.

In the following we will compare the mean-field values for $\varepsilon_{0,8}^{\text {crit }}$ for Eqs. (41), (47), (55) with the large- $N_{f}$-results.

\subsection{Critical meson masses in the large- $N_{f}$-expansion}

The $S U(3)$-symmetric case. When $\varepsilon_{8}=0$ and $\varepsilon_{0}$ is slowly increased from 0 to $\sim 2.5 \cdot 10^{-4}[\mathrm{GeV}]^{3}$, we observe a weakening of the first order transition as is seen in Fig. 5. For the critical field strength we find

$$
\varepsilon_{0}^{\text {crit }} \leq(3 \pm 0.5) \cdot 10^{-4}[\mathrm{GeV}]^{3}
$$

The value for $\varepsilon_{0}^{\text {crit }}$ induces a pseudoscalar meson mass of 51 [MeV] or quark masses of $m_{u, d}^{\text {crit }}=m_{s}^{\text {crit }}=0.9 \pm 0.14[\mathrm{MeV}]$, such that

$$
m_{u, d, s}^{c r i t} / m_{u, d, s} \sim 0.08 \pm 0.01
$$

Within the errors the result for $\varepsilon_{0}^{\text {crit }}$ is of the same order of magnitude as the mean-field value. The uncertainty in our result is $\Delta \varepsilon_{0}^{\text {crit }} \leq 0.5 \cdot 10^{-4}[\mathrm{GeV}]^{3}$. For 
Figure 5: The light quark condensate normalized to its value at zero temperature $\langle\bar{q} q\rangle_{T} /\langle\bar{q} q\rangle_{0}$ as a function of $T$ in the $S U(3)$-symmetric case. The weakening of the first order transition is obvious, when $\varepsilon_{0}[\mathrm{GeV}]^{3}$ is varied between $\varepsilon_{0}=0(\times), 2$. $10^{-4}(\diamond), 2.5 \cdot 10^{-4}(+)$ and $6.6 \cdot 10^{-4}(\square)$.

$\varepsilon_{0}=2.5 \cdot 10^{-4}[\mathrm{GeV}]^{3}$ the transition could be clearly identified as first order from the $\langle\bar{q} q\rangle_{T} /\langle\bar{q} q\rangle_{0}(T)$-curves. For $\varepsilon_{0}=3 \cdot 10^{-4}[\mathrm{GeV}]$ it is a crossover. We could have further improved the accuracy of $\varepsilon_{0}^{c r i t}$ by measuring data for $\langle\bar{q} q\rangle_{T}(T)$ in the intermediate $\varepsilon_{0}$-range. On the other hand such an improvement is anyway limited by the well-known fact that it is in general hard to disentangle a very weak first order transition from a rapid crossover phenomenon.

The weakening of the first order transition is also revealed in the barrier height of the effective potential between the coexisting minima at $T_{c}$. The barrier decreases from $1.4 \cdot 10^{-4}\left[\mathrm{GeV} / \mathrm{fm}^{3}\right]$ in the chiral limit to $2.1 \cdot 10^{-6}\left[\mathrm{GeV} / \mathrm{fm}^{3}\right]$ for $\varepsilon_{0}=2.0$. $10^{-4}[\mathrm{GeV}]^{3}, \varepsilon_{8}=0[\mathrm{GeV}]^{3}$, the largest value for which a first order transition could be identified from the shape of the effective potential, cf. Fig. 6. A comparison between Figs. 5 and 6 shows the ambiguity in identifying a very weak 1st order 
transition. Fig. 5 suggests a weak 1 st order transition for $\varepsilon_{0}=2.5 \cdot 10^{-4}[\mathrm{GeV}]^{3}$ with $T_{c} \sim 181[\mathrm{MeV}]$, but no barrier is visible between the two coexisting condensate values at the same temperature and the same $\varepsilon_{0}$-value in Fig. 6. Accordingly a precise determination of $T_{c}$ is hard in case of a weak first order transition such that we estimate for the error in $T_{c} \Delta T_{c}= \pm 2[\mathrm{MeV}]$. Fig. 6 also admits an estimate of the error in finding $\left\langle\sigma_{0,8}\right\rangle$ in the transition/crossover region if the effective potential is very flat, which we have mentioned in section 3 . In the chiral limit we have $\Delta\left\langle\sigma_{0}\right\rangle \sim 1.3 \cdot 10^{-3}[\mathrm{GeV}]$, for $\varepsilon_{0}=2 \cdot 10^{-4}$ and $2.5 \cdot 10^{-4}$ the error is estimated as $4.3 \cdot 10^{-3}[\mathrm{GeV}]$. Later we assume $\Delta\left\langle\sigma_{0}\right\rangle \sim \Delta\left\langle\sigma_{8}\right\rangle$.

Figure 6: Decrease of the barrier height of the effective potential $\hat{U}_{\text {eff }}$ for $\varepsilon_{0}=0$ at $T \sim 176[\mathrm{MeV}]$ (solid curve), $\varepsilon_{0}=2 \cdot 10^{-4}[\mathrm{GeV}]^{3}$ at $T \sim 180[\mathrm{MeV}]^{3}$ (dashed curve), $\varepsilon_{0}=2.5 \cdot 10^{-4}[\mathrm{GeV}]^{3}$ for $T \sim 181[\mathrm{MeV}]$ (determined from the corresponding condensate curve of Fig. 5) (dotted curve) in the $S U(3)$-symmetric case.

$T_{c}(m)$-dependence in the $S U(3)$-symmetric case. For simplicity we restrict the study of the mass dependence of $T_{c}$ in the first order transition region to the $S U(3)$ symmetric case. In Fig. 7 we see an increase of $T_{c}$ with the current quark mass 
$\hat{m}[\mathrm{MeV}]$. For realistic mass values of $m_{\pi}=129.3[\mathrm{MeV}], m_{K}=490.7[\mathrm{MeV}]$,

Figure 7: The critical temperature $T_{c}$ as a function of the average light quark mass $\hat{m}[\mathrm{MeV}]$ in the $S U(3)$-symmetric case. For further explanations see the text.

$m_{\eta}=544.7[\mathrm{MeV}]$ (not depicted in Fig.7), the rapid crossover sets in at $T \sim 181.5$ $[\mathrm{MeV}]$ and becomes slow at $\sim 192.6[\mathrm{MeV}]$. We associate a critical temperature " $T_{c}$ " $\sim 187 \pm 0.5[\mathrm{MeV}]$ to this crossover phenomenon for comparison with extrapolated values of $T_{c}$ in chiral perturbation theory (in our case $T_{c}$ is localized as the point of inflection in the crossover curve). Thus $T_{c}$ has increased by $5.3 \%$ compared to the chiral limit. This result is in agreement with the estimate of Leutwyler [29], who predicts $\Delta T_{c} / T_{c} \sim 5 \%$ if realistic pion masses are substituted for the chiral limit with $m_{\pi}=0$. When the critical temperature is extrapolated in the framework of chiral perturbation theory, the inclusion of finite quark masses delays the melting of $\langle\bar{q} q\rangle$ by $\Delta T \sim 20[\mathrm{MeV}]$ [4], while the inclusion of heavier mesons in a dilute gas approximation has the opposite effect. At finite quark masses it accelerates the melting from $T_{c} \sim 240[\mathrm{MeV}]$ to $190[\mathrm{MeV}]$. The delay in the melting due to finite quark masses is intimately related to the size of the latent heat. The relation is 
revealed in the derivation of a Clausius-Clapeyron-equation for QCD, cf. 29]. Thus the $T_{c}(m)$-dependence is conclusive for the strength of a first order chiral transition.

The case of realistic mass splitting, $\varepsilon_{0}^{\text {crit }} / \varepsilon_{8}^{\text {crit }}=0.77$. For the realistic mass splitting induced by $m_{s} / m_{u, d}=18.2$ we find for the critical field strengths in large$N_{f}$

$$
\varepsilon_{0}^{\text {crit }} \leq(7 \pm 2) \cdot 10^{-3}[\mathrm{GeV}]^{3}, \quad \varepsilon_{8}^{\text {crit }} \leq(-9.09 \pm 2.6) \cdot 10^{-3}[\mathrm{GeV}]^{3}
$$

with $m_{\pi}=68.7, m_{K}=276.8, m_{\eta}=313.4, m_{\eta^{\prime}}=928.6, m_{\sigma_{\pi}}=887.9, m_{\sigma_{K}}=$ 890.9, $m_{\sigma_{\eta}}=924.3, m_{\sigma_{\eta^{\prime}}}=698.2$ (all masses in units of $[\mathrm{MeV}]$ ) or an average pseudoscalar octet mass of $\left\langle m_{p s}\right\rangle=203[\mathrm{MeV}]$. The critical values for the current quark masses are $m_{u, d}^{\text {crit }}=2.96 \pm 0.85[\mathrm{MeV}]$ and $m_{s}^{\text {crit }}=54 \pm 15.4[\mathrm{MeV}]$. The reason why we give an upper bound on $\varepsilon_{0,8}^{c r i t}$ rather than precise values for the first order transition boundary is the same as in the $S U(3)$-symmetric case. The bound on $\varepsilon_{0,8}^{\text {crit }}$ could still be improved by measuring data between $\varepsilon_{0}=4 \cdot 10^{-3}[\mathrm{GeV}]^{3}$, $\varepsilon_{8}=-5.2 \cdot 10^{-3}[\mathrm{GeV}]^{3}$, where the transition is still of first order, and the above values for $\varepsilon_{0,8}^{\text {crit }}$. It should be remarked that only a rather fine resolution of the $\langle\bar{q} q\rangle /\langle\bar{q} q\rangle_{0}(T)$ curve in steps of $\Delta T \leq 0.1[\mathrm{MeV}]$ has revealed the true 1st order nature of the transition for $\varepsilon_{0}=5 \cdot 10^{-3}$ and $\varepsilon_{8}=-6.5 \cdot 10^{-3}[\mathrm{GeV}]^{3}$ due to the small gap in the condensate. The resolution in units of $\Delta T=1[\mathrm{MeV}]$ had suggested a smooth crossover behaviour already for these smaller values of $\varepsilon_{0,8}$.

Thus we see that also in a large $N_{f}$-expansion tiny values for the quark masses are sufficient to weaken the chiral transition and turn it finally into a crossover phenomenon. Similarly tiny current quark masses are sufficient to eliminate the chiral transition in an $S U(3)$-Nambu-Jona-Lasinio model as a function of temperature and nuclear density [30].

The large- $N_{f}$-results for $\varepsilon_{0,8}^{\text {crit }}$ are clearly above the mean-field values of Eq. (47). The result is plausible as our saddle-point approximation goes beyond the mean-field calculation. The leading term in a $1 / N_{f}$-expansion corresponds to the summation of a class of diagrams, called "super-daisies" 31]. Super-daisies have been summed up by Dolan and Jackiw [23] to circumvent the IR-divergence problem in an $N$-component $\phi^{4}$-theory. Our application of the $1 / N_{F}$-method to the linear sigma-model has been similar in spirit. Certainly we cannot claim that the fluctuations we have included so far are representative for all fluctuations. In fact, the classical cubic term of our potential may still dominate the driving mechanism for the first order transition below the critical field strengths. Only the lattice calculation includes all fluctuations by simulating the full partition function at once (at least in principle). This 
may explain, why a discrepancy of $\approx 0.25$ remains between the large- $N_{f}$-ratio and the lattice result for $m_{u, d}^{c r i t} / m_{u, d}$. We should keep in mind, however, that the lattice result itself is not yet reliable, as we have argued in section 1.

The next question which arises in a comparison with the mean-field calculation concerns the critical renormalized masses which should vanish for the critical field strengths at a second order transition. In the mean-field calculation $m_{\sigma_{\eta^{\prime}}}^{2}$ vanishes by construction for the set of critical parameters, and it is easily checked that $m_{\sigma_{\eta^{\prime}}}$ is the only mass that vanishes at criticality. Our value for $m_{\sigma_{\eta^{\prime}}}=749.5[\mathrm{MeV}]$ is the tree level input mass at zero temperature, which need not vanish. The effective masses $X_{Q}$ are temperature dependent. They slowly de/increase with $T$ as we will see in section 6 . We have mentioned in the discussion of the chiral limit (cf. section 4) that $X_{Q}, Q=1 \ldots 4$ violate Goldstone's theorem, if they coincide with a dynamical mass in our approximation. Similarly it is here not obvious that one of these masses should induce an infinite correlation length as $T$ approaches $T_{c}$. A careful renormalization prescription should be imposed to identify the renormalized mass(es) that go to zero for critical external fields. It would further allow an identification of the universality class of the $S U(3) \times S U(3)$-linear sigma-model for critical parameters $\mu_{0}^{2}, f_{1}, f_{2}, g, \varepsilon_{0}, \varepsilon_{8}$. We will investigate these questions in a forthcoming work.

Critical meson masses for $m_{s}=0$. Here the large- $N_{f}$-results are even somewhat smaller than the mean-field results (cf. Eqs. (55)). We find

$$
\varepsilon_{0}^{c r i t}=(4 \pm 1) \cdot 10^{-4}[\mathrm{GeV}]^{3} \quad \varepsilon_{8}^{c r i t}=(3.06 \pm 0.76) \cdot 10^{-4}[\mathrm{GeV}]^{3}
$$

The associated average pseudoscalar octet mass is $\left\langle m_{p s}\right\rangle=57.2 \pm 28.8[\mathrm{MeV}]$, and $m_{u, d}=1.7 \pm 0.4[\mathrm{MeV}]$, while $m_{s}=0$ by construction.

The Columbia plot (cf. Fig.1) has suggested a concave shape of the first order transition boundary. The three critical masses in mean-field are compatible with such a shape in an $\left(m_{\pi}, m_{K}\right)$ - or an $\left(\hat{m}, m_{s}\right)$-diagram, although one should keep in mind the large error bars due to the ambiguity in the tree-level parametrization of the sigma-model and the sensitive dependence of the boundary on the $\sigma_{\eta^{\left({ }^{\prime}\right)}}$-mass input. The three critical masses in large- $N_{f}$ do not confirm the conjectural concave shape. Only for a realistic ratio of $\varepsilon_{0} / \varepsilon_{8}$ the large- $N_{f}$-result lies clearly above the mean-field value for the critical masses. The error here is at least as large as in the mean-field case. The error could have been further reduced, but the remaining size would reflect the difficulty in disentangling a very weak first order transition from a rapid crossover phenomenon as we have mentioned above.

In the next section we will see the change in the crossover behaviour, as $\varepsilon_{0}, \varepsilon_{8}$ 
are further increased to induce realistic mass values.

\section{The realistic mass point}

If we use for $\mu_{0}^{2}, f_{1}, f_{2}, g$ the values of the chiral limit and choose $\varepsilon_{0}=0.0265[\mathrm{GeV}]^{3}$, $\varepsilon_{8}=-0.0345[\mathrm{GeV}]^{3}$, Eqs. (10 11) lead to (pseudo)scalar meson masses which are listed in Table 1. A comparison to the experimental values shows reasonable agreement for the pseudoscalar mesons. Therefore we call this point the "realistic" mass point. The experimental values which are associated to the scalar meson masses depend on the identification, which is indicated in a separate row of Table 1 . The mass splitting between $K_{0}^{*}$ and $a_{0}$ comes out too small in our case. We could have further optimized our choice of $\varepsilon_{0}$ and $\varepsilon_{8}$ to improve the agreement with the experimental mass values, but such an optimization should be inconsequential for our results.

Table 1: Tree level parametrization of the $S U(3) \times S U(3)$ linear sigma-model for the realistic mass point

Input

\begin{tabular}{|l|l|l|l|l|l|l|}
\hline$\mu_{0}^{2}[\mathrm{GeV}]^{2}$ & $f_{1}$ & $f_{2}$ & $g[\mathrm{MeV}]$ & $f_{\pi}[\mathrm{MeV}]$ & $\varepsilon_{0}[\mathrm{GeV}]^{3}$ & $\varepsilon_{8}[\mathrm{GeV}]^{3}$ \\
\hline $5.96 \cdot 10^{-2}$ & 4.17 & 4.48 & $-1,812.0$ & 94 & 0.0265 & -0.0345 \\
\hline
\end{tabular}

Output (all masses are understood in units of $[\mathrm{MeV}]$ )

\begin{tabular}{|l|l|l|l|l|l|l|l|l|}
\hline & $m_{\pi}$ & $m_{K}$ & $m_{\eta}$ & $m_{\eta^{\prime}}$ & $m_{\sigma_{\pi}}$ & $m_{\sigma_{K}}$ & $m_{\sigma_{\eta}}$ & $m_{\sigma_{\eta^{\prime}}}$ \\
\hline $\begin{array}{l}\text { real. } \\
\text { mass } \\
\text { point }\end{array}$ & 129.3 & 490.7 & 544.7 & 1045.5 & 1011.6 & 1031.2 & 1198.0 & 749.5 \\
\hline $\begin{array}{l}\text { exp. } \\
\text { mass } \\
\text { values }\end{array}$ & 138.0 & 495.7 & 547.5 & 957.8 & $\begin{array}{l}980 \text { if } \\
\sigma_{\pi} \equiv \\
a_{0}\end{array}$ & $\begin{array}{l}1322.0 \text { if } \\
\sigma_{K} \equiv \\
K_{0}^{*}\end{array}$ & $\begin{array}{l}1476.0 \text { if } \\
\sigma_{\eta} \equiv \\
f_{0}(1476)\end{array}$ & $\begin{array}{l}975 \text { if } \\
\sigma_{\eta^{\prime}} \equiv \\
f_{0}(975)\end{array}$ \\
\hline
\end{tabular}

Crossover in the condensates. The crossover behaviour for the normalized light and strange quark condensates are displayed in Fig. 8. The rapid crossover leads to a decrease of $\sim 50 \%$ of the condensate at zero temperature $\langle\bar{q} q\rangle_{0}$ in $\langle\bar{q} q\rangle_{T}$ over a temperature interval $\Delta T \sim 10[\mathrm{MeV}]$, while $\langle\bar{s} s\rangle_{T}$ stays remarkably constant up to $T \sim 197[\mathrm{MeV}]$ where it starts to decrease rather slowly. The physical reason is obvious. It is harder to excite mesons with strange quarks than with light quarks. 
Figure 8: Light $(\langle\bar{q} q\rangle)$ and strange $(\langle\bar{s} s\rangle)$ quark condensates normalized to their corresponding values at zero temperature as a function of temperature. The crossover behaviour is most rapid between $181.5 \leq T \leq 192.6[\mathrm{MeV}]$.

The same qualitative behaviour of $\langle\bar{s} s\rangle_{T}$ has been noticed by Hatsuda and Kunihiro 32] in a $U_{N_{f}}(3)$-version of the Nambu-Jona-Lasinio model. Also the location of the crossover region in the NJL-model is around $T \sim 200[\mathrm{MeV}]$.

We have indicated the error bars only in the crossover region where they are largest. As outlined in section 3, their main sources are the uncertainty in precisely locating the saddle point value $s a d^{*}$ and the minima $\left\langle\sigma_{0}\right\rangle,\left\langle\sigma_{8}\right\rangle$ of $\hat{U}_{\text {eff }}$. When the errors in the current quark masses are added, which enter Eq. (14), we obtain $\Delta\langle\bar{q} q\rangle \sim$ $3.1 \cdot 10^{-3}[\mathrm{GeV}]^{3}, \Delta\langle\bar{s} s\rangle \sim 6.7 \cdot 10^{-3}[\mathrm{GeV}]^{3}$ at $T=180[\mathrm{MeV}]$ in the transition region. In Fig. 8 we have indicated only the numerical errors, the contribution from the current quark masses has been left out. Compared to critical meson masses $\left(\varepsilon_{0}^{\text {crit }}=7 \cdot 10^{-3}[\mathrm{GeV}]^{3}, \varepsilon_{8}^{\text {crit }}=-9.1 \cdot 10^{-3}[\mathrm{GeV}]^{3}\right)$, the crossover happens over a larger temperature interval. We find $\Delta\left[\langle\bar{q} q\rangle_{T} /\langle\bar{q} q\rangle_{0}\right]^{\text {real }} / \Delta\left[\langle\bar{q} q\rangle_{T} /\langle\bar{q} q\rangle_{0}\right]^{\text {crit }} \sim 52 \%$ if $\Delta\left[\langle\bar{q} q\rangle_{T} /\langle\bar{q} q\rangle_{0}\right]$ denotes the normalized condensate change per $1[\mathrm{MeV}]$ temperature 
interval in the rapid part of the crossover region. Nevertheless the crossover in the quark condensate $\langle\bar{q} q\rangle$ seems to be sharp even for realistic masses. Such a rapid change may lead to visible changes in hadron masses which depend on temperature and condensates. It could be manifest in hadronic or dilepton spectra in heavy-ion experiments.

Figure 9: Contour plot of $\hat{U}_{\text {eff }}\left(\sigma_{0}, \sigma_{8}\right)$ in a projection on the $\left(\sigma_{0}, \sigma_{8}\right)$-plane for four temperatures. In Fig. 9a) $(T=10[\mathrm{MeV}])$ we see two separated minima (indicated with crosses), one unphysical with $\sigma_{0}<0, \sigma_{8}<0$ and the absolute physcial minimum with $\sigma_{0}>0, \sigma_{8}<0$. In the crossover region at $T=190[\mathrm{MeV}]$ (Fig. 9b)) both minima lie in the physical sector with $\sigma_{0}>0, \sigma_{8}<0$. The minima coincide for temperatures $\geq 200[\mathrm{MeV}]$ (Fig. 9c) with $T=200[\mathrm{MeV}]$ ). For increasing temperature the one remaining minimum moves inwards, but stays away from zero due to $\varepsilon_{0,8} \neq 0$, as is seen in Fig. 9d) for $T=350[\mathrm{MeV}]$.

The crossover behaviour in the condensates is also revealed in two-dimensional contour plots of the effective potential $\hat{U}_{e f f}\left(\sigma_{0}, \sigma_{8} ; s a d^{*}\right)$. In Fig. 9 we show equipotential lines for four temperatures in a projection of $\hat{U}_{\text {eff }}$ on the $\left(\sigma_{0}, \sigma_{8}\right)$-plane. In 
Fig. 9a) we see two separated local minima for $T=10[\mathrm{MeV}]$, one in the sector $\sigma_{0}<0, \sigma_{8}<0$ and another one in the physical sector $\sigma_{0} \geq 0, \sigma_{8} \leq 0$. (In the chiral limit we would find three minima due to $\varepsilon_{0}=0=\varepsilon_{8}$.) In the crossover region at $T=190[\mathrm{MeV}]$ (Fig. 9b)) we still have two separated minima, where the left one is a local minimum and the right one is the absolute minimum of $\hat{U}_{\text {eff }}$. Now both minima lie in the physical sector. At $T=200[\mathrm{MeV}]$ (Fig. 9c)) the minima have merged in a single one. As the temperature is further increased, the absolute minimum moves inwards, but stays away from zero due to the explicit symmetry breaking fields $\varepsilon_{0}$ and $\varepsilon_{8}$ (cf. Fig. 9d) for $T=350[\mathrm{MeV}]$ ). The elliptical shape of the contour plot reflects the violation of spherical symmetry in $\left(\sigma_{0}, \sigma_{8}\right)$-space due to the explicit symmetry breaking, the cubic term in the classical potential and the thermal part. As the thermal part gets more dominant at higher temperatures, its high-temperature limit changes the elliptical shape to an approximately spherical symmetric shape. The two minima below $200[\mathrm{MeV}]$ may be easily misinterpreted as the coexistence of the symmetric and the chiral phase 18, if one does not realize that the minimum $\left(\sigma_{0}, \sigma_{8}\right)$ on the r.h.s. of Fig. 9b) stays the absolute minimum for all temperatures.

Thermodynamics. Further characteristics of the crossover phenomenon are the variations in the energy and entropy densities over the temperature interval in the crossover region. At this place one should recall the very definition of a first order phase transition. At the transition point at least one of the first derivatives of a suitable thermodynamic potential should behave discontinuously in the infinite volume limit. Thus a crossover in the condensates in general does not exclude a finite gap in the energy or entropy densities. In Fig. 10 we have plotted $s / T^{3}, \varepsilon / T^{4}$, and $p / T^{4}$ as calculated according to Eqs. (2729). The data points are strongly fluctuating within a range which is indicated by the error bars for the entropy curve. The errors for the energy density are of similar size. Errors enter via the effective masses $X_{Q}^{2}$, which depend on $\left\langle\sigma_{0}\right\rangle,\left\langle\sigma_{8}\right\rangle$ and $s a d^{*}$. We have used for $\Delta\left\langle\sigma_{0}\right\rangle \sim$ $(1.0-3.0) \cdot 10^{-3}[\mathrm{GeV}]=\Delta\left\langle\sigma_{8}\right\rangle$ and $\Delta\left\langle s a d^{*}\right\rangle \sim 0.11[\mathrm{GeV}]^{2}$ in the crossover region between $T=182-193[\mathrm{MeV}]$. The pressure behaves continuously as a function of $T$ if it is calculated as $p=\left(-\hat{U}_{\text {eff }}\right)$. There is only a change of slope in the critical crossover region. A direct calculation of $p$ with an integral expression pretends a discontinuity. The $p / T^{4}$-curve in Fig. 10 is obtained from $\left(p=-\hat{U}_{\text {eff }}\right)$. As change in the entropy density we find from the actually measured data

$$
T \cdot \Delta s \leq 0.16\left[\mathrm{GeV} / \mathrm{fm}^{3}\right]
$$

in a temperature interval $181.5 \leq T \leq 192.6[\mathrm{MeV}]$, where $T \cdot \Delta s$ is calculated as 
Figure 10: Entropy density $s$ over $T^{3}$, energy density $\varepsilon$ over $T^{4}$ and pressure $p$ over $T^{4}$ in the large $N_{f}$-expansion for the realistic mass point. Errors are only indicated for $s / T^{3}$.

$T_{2} \cdot s\left(T_{2}\right)-T_{1} \cdot s\left(T_{1}\right)$. As rapid change in the energy density we find

$$
\Delta \varepsilon \leq 0.13\left[\mathrm{GeV} / \mathrm{fm}^{3}\right]
$$

or $\Delta \varepsilon / T_{c}^{4} \equiv \varepsilon\left(T_{2}=192.6[\mathrm{MeV}]\right) / T_{2}^{4}-\varepsilon\left(T_{1}=181.5[\mathrm{MeV}]\right) / T_{1}^{4}=0.29$ over the same temperature range. For comparison we mention that the gap in the gluonic energy density $\Delta \varepsilon_{g}$ in a pure $S U(3)$ gauge theory leads to [33, 34]

$$
\frac{\Delta \varepsilon_{g}}{T_{c}^{4}}= \begin{cases}2.44 \pm 0.24, & \text { for } N_{\tau}=4 \\ 1.80 \pm 0.18, & \text { for } N_{\tau}=6\end{cases}
$$

where $N_{\tau}$ refers to the lattice extension in time direction. These values are by an order of magnitude larger than our value for the mesonic contribution $\Delta \varepsilon / T_{c}^{4} \sim 0.25$, defined as indicated above. The decrease of $\Delta \varepsilon_{g} / T_{c}^{4}$ under an increase of the time extension $N_{\tau}$ indicates strong finite size effects. Going to larger lattices this tendency may continue and further reduce the latent heat, but it also gives us a warning. The 
contribution of $\Delta \varepsilon_{g}$ to the total energy gap may be superimposed on the slow change of $\varepsilon$ that we have found in the crossover region and make the crossover in the total energy density more rapid. If the size of the errors in $s a d^{*},\left\langle\sigma_{0}\right\rangle,\left\langle\sigma_{8}\right\rangle$ is assumed as above, $s$ could vary in the crossover region between $T_{1}$ and $T_{2}$ like

$$
s\left(T_{2}\right) \cdot T_{2}-s\left(T_{1}\right) \cdot T_{1} \leq 0.18\left[\mathrm{GeV} / \mathrm{fm}^{3}\right],
$$

where the resulting error $\Delta\left(s / T^{3}\right)$ has been estimated as \pm 0.46 , cf. Fig. 10. In an infinitesimally small temperature interval such a gap in $s$ would lead to a finite latent heat of $\leq 0.2\left[\mathrm{GeV} / \mathrm{fm}^{3}\right]$. Thus Eq. (63) gives a loose upper bound on the latent heat which could be compatible with our data due to the large errors in the crossover region. The bound comes out even smaller if the error of 0.46 is interpreted as $\Delta s / T_{c}^{3}$ with " $T_{c}$ " $=187[\mathrm{MeV}]$. It leads to $\Delta L=T_{c} \cdot \Delta s \leq 0.074\left[\mathrm{GeV} / \mathrm{fm}^{3}\right]$. Both bounds are even smaller than Leutwyler's value of $0.4\left[\mathrm{GeV} / \mathrm{fm}^{3}\right]$ for $T \cdot \Delta s$ [29], which has been obtained from a Clausius-Clapeyron equation in the framework of chiral perturbation theory. The small size of the latent heat is finally a consequence of the sensitivity of $T_{c}$ to the inclusion of finite quark masses.

In view of heavy-ion experiments there need not be a latent heat in the strict sense, which occurs over a time period during the phase conversion, where the temperature stays exactly constant. Multiplicity fluctuations in rapidity distributions of charged particles are also induced if $\Delta s$ is sufficiently large over a small, but finite temperature interval (say of the order of $\sim 10[\mathrm{MeV}]$ ). In van Hove's formulation [15] the physical conditions of a first order transition are identical to those of a rapid crossover phenomenon.

Clausius-Clapeyron equations relate the discontinuities in the condensate and the entropy/energy densities. Although they strictly apply to first order transitions in the form of Eqs. (64 65) below [29, we have tested the relations for our rapid crossover phenomenon in two forms. The first one is

$$
\left.\operatorname{disc}\langle\bar{q} q\rangle_{T}\right|_{\text {r.c. }}=\left.\left.\frac{\Delta T_{c}}{\Delta \hat{m}}\right|_{\text {r.c. }} \operatorname{disc} s\right|_{\text {r.c. }}
$$

where disc... $\left.\right|_{\text {r.c. }}$ refers to the rapid change ("discontinuity") in the crossover region and $\Delta T_{c}$ is the change in " $T_{c}$ " under a variation $\Delta \hat{m}$ of the current light quark masses. The second version is

$$
\left.\frac{\Delta T_{c}}{T_{c}}\right|_{r . c .}=\left|\frac{\operatorname{disc}\langle\bar{q} q\rangle_{T}}{\langle\bar{q} q\rangle_{0}}\right|_{\text {r.c. }}\left|\frac{f_{\pi}^{2} m_{\pi}^{2}}{\Delta \varepsilon}\right|_{\text {r.c. }} .
$$

In Eq. (64) we use $\Delta T_{c} / \Delta \hat{m}=(0.187-0.178) /(0.011), \Delta s=0.0061[\mathrm{GeV}]^{3}$, $\operatorname{disc}\langle\bar{q} q\rangle_{T}=0.5 \cdot(0.22)^{3}$, and obtain 0.005 for the 1.h.s. and for the r.h.s. of Eq. (64). 
In Eq. (65) we have $\Delta T_{c}$ as above, $T_{c}=178[\mathrm{MeV}], \operatorname{disc}\langle\bar{q} q\rangle_{T} /\langle\bar{q} q\rangle_{0}=\langle\bar{q} q\rangle_{T_{1}} /\langle\bar{q} q\rangle_{0}-$ $\langle\bar{q} q\rangle_{T_{2}} /\langle\bar{q} q\rangle_{0}=0.5, f_{\pi}=94[\mathrm{MeV}], m_{\pi}=129[\mathrm{MeV}]$ (the value of our realistic mass point), and $\Delta \varepsilon=0.001[\mathrm{GeV}]^{4}$. This way we obtain for the r.h.s. $7 \%$ and for the l.h.s. $5 \%$ in Eq. (65)). The agreement of the order of magnitude on both sides indicates that the crossover phenomenon is still rapid enough to satisfy analogous relations to a first order transition in the strict sense.

The same relations have been checked for the first order transition in the chiral limit. Eq.s (64),(65) with the appropriate chiral input data predict for $\Delta \varepsilon / T_{c}^{4} \sim$ $1.0 \pm 0.2 \sim \Delta s / T_{c}^{3}$. The actually measured gap $\Delta s / T_{c}^{3}$ in chiral thermodynamics comes out as $0.5 \pm 0.2$. An error of 0.2 is easily induced by an error of $10[\mathrm{MeV}]$ in the condensates $\left\langle\sigma_{0}\right\rangle,\left\langle\sigma_{8}\right\rangle$ at the phase transition. Thus the relations are approximately satisfied also in the chiral limit within the relatively large errors.

We have further analyzed the contribution of the strange (pseudo)scalar mesons $K$ and $\sigma_{K}$ to the total energy density $\varepsilon$. Their contribution can be completely neglected below $40[\mathrm{MeV}]$. It increases with temperature to $\sim 31 \%$ in the crossover region around $T=187[\mathrm{MeV}]$. After the crossover the tendency continues, but is no longer conclusive for us due to the lack of quark degrees of freedom in the chiral symmetric phase.

The strangeness content of the plasma has been estimated in a lattice simulation 35] with light quark masses of $m_{u} / T=m_{d} / T=0.05$ and one heavier quark mass of $m_{s} / T=1.0$. In the transition region one finds for the ratio of the fermionic energy densities $\varepsilon_{F}\left(m_{s} / T=1\right) / \varepsilon_{F}\left(m_{u} / T=0.05\right) \simeq 0.5$. The good agreement with our ratio of mesonic contributions from strange and non-strange (pseudo)scalar mesons $(\varepsilon$ (strange mesons $) / \varepsilon$ (non-strange mesons $)) \sim 0.45$ may be accidental, because the lattice estimate is based on perturbative relations for the energy density. A fully nonperturbative lattice calculation along the lines of Engels et al. [33] in a pure $S U(3)$ gauge theory is still outstanding if fermions are included. Nevertheless, it should be mentioned that an extrapolation for fermionic energy densities $\varepsilon_{F}(m / T=1)$ and $\varepsilon_{F}(m / T=0)$ from the plasma phase to the transition region under the assumption of ideal fermion gases leads to $\varepsilon_{F}(m / T=1) / \varepsilon_{F}(m / T=0)=0.88$ [36], which is twice the amount of our ratio for mesons in the transition region. This may be taken as an indication that the strangeness contribution to $\varepsilon$ around $T_{c}$ cannot be derived from an underlying ideal gas of quarks of $m_{s}$ and $m_{u, d}$-quarks.

Temperature dependence of effective masses. Fig. 11 displays the temperature dependence of the effective masses $X_{Q}, Q=1, \ldots, 8$ up to temperatures of $300[\mathrm{MeV}]$. We have found a degeneracy of all (pseudo)scalar meson masses at higher tempera- 
Figure 11: Temperature dependence of the effective mass squares $X_{Q}, Q=1, \ldots, 8$ for realistic meson masses. From bottom to top: $X_{\pi}, X_{K}, X_{\eta}, X_{\sigma_{\eta^{\prime}}}, X_{\sigma_{\pi}}, X_{\sigma_{K}}, X_{\eta^{\prime}}, X_{\sigma_{\eta}}$.

tures $(T \geq 400[\mathrm{MeV}])$, where the sigma-model fails to describe the plasma phase. Immediately above the rapid crossover region chiral symmetry is partially restored. The restoration in strange and non-strange sectors is achieved quite differently. We find an approximate degeneracy between $X_{\pi}$ and $X_{\sigma_{\eta^{\prime}}}, X_{K}, X_{\eta}$ and $X_{\sigma_{K}}, X_{\eta^{\prime}}$ and $X_{\sigma_{\pi}}$, while $X_{\sigma_{\eta}}$ becomes degenerate with other masses only at $T \sim 400[\mathrm{MeV}]$. The $\sigma_{\pi}$-mass never meets the $\pi$-mass, because the $U_{A}(1)$-anomaly term (proportional to $g$ ) enters the mass formulas with opposite sign. From lattice results it is very likely that other modes than $X_{\pi}, X_{\sigma_{\eta^{\prime}}}$ dissolve in their constituents and cease to exist in the chiral symmetric phase. Coming from the broken phase we notice that the effective mass $X_{\sigma_{\eta^{\prime}}}$ drops to a remarkably small value in the crossover region $\left(X_{\sigma_{\eta^{\prime}}} \sim\left(X_{\pi}\right.\right.$ at $T=0) \sim 130[\mathrm{MeV}]$ ), while $X_{\pi}$ is almost zero. Furthermore, we have displayed the errors in $X_{\pi}$ and $X_{\sigma_{\eta^{\prime}}}$ in the transition region. The errors have increased with $T$ to these values due to the contribution of $\Delta \operatorname{sad}(T)$. For $X_{\pi}$ the error is particularly large in the crossover region, since the error $\Delta X_{\pi}$ is proportional to $1 / X_{\pi}$ and $X_{\pi}$ is 
almost zero in this region. Before we jump to conclusions about measurable effects due to tiny $\sigma_{\eta^{\prime}}$ and $\pi$-masses in the crossover region, we should clarify the precise physical meaning of the effective masses $X_{Q}$. We recall that the main contribution to the temperature dependence of $X_{Q}$ comes from the saddle point contribution $\operatorname{sad}^{*}(T)$, which is just the leading term in the $1 / N_{f}$-expansion.

\section{$7 \quad$ Summary of results and conclusions}

In agreement with the general expectation results of the high- $T$-expansion are qualitatively correct, but fail quantitatively. The critical temperature in the chiral limit deviates by $\sim 85[\mathrm{MeV}]$ from $T_{c}$ in the numerical evaluation, which is applicable also in the transition/crossover region. The crossover region for realistic meson masses is shifted by roughly $80[\mathrm{MeV}]$ between the high- $T$ - and the numerical results. In our model the high- $T$-expansion practically never reproduces the numerical results in a quantitative way. While the meson condensate $\left\langle\sigma_{0}\right\rangle$ in the realistic mass case has dropped to values $\leq 2[\mathrm{MeV}]$ around $T \sim 400[\mathrm{MeV}]$ in the high- $T$-expansion, it is still larger than $30[\mathrm{MeV}]$ at this temperature in the numerical calculation. The temperature lies already far outside the applicability range of the model.

The crossover in the light quark condensate looks still rapid for realistic meson masses $\left(\Delta\left(\langle\bar{q} q\rangle_{T}\right) \sim 50 \%\right.$ of $\langle\bar{q} q\rangle_{0}$ in a temperature interval of $10[\mathrm{MeV}]$ in the crossover region). Less rapid look the variations in the energy and entropy densities between $181.5[\mathrm{MeV}] \leq T \leq 192.6[\mathrm{MeV}]$. We find $\Delta \varepsilon \sim 0.13 \pm 0.02\left[\mathrm{GeV} / \mathrm{fm}^{3}\right]$ and $T \cdot \Delta_{s} \sim 0.16 \pm 0.02\left[\mathrm{GeV} / \mathrm{fm}^{3}\right]$. As a loose bound on the latent heat we obtain $0.2\left[\mathrm{GeV} / \mathrm{fm}^{3}\right]$, as a more stringent one $0.1\left[\mathrm{GeV} / \mathrm{fm}^{3}\right]$, if the change in $\varepsilon$ and $s$ would occur over an arbitrarily small temperature interval. The main contributions to the numerical errors which prevent us from a unique interpretation of the transition/crossover region come from the uncertainties in the saddle point value and the minima $\left\langle\sigma_{0}\right\rangle,\left\langle\sigma_{8}\right\rangle$ of $\hat{U}_{e f f}$.

For temperatures above $116[\mathrm{MeV}]$ the saddle point variable gives a positive contribution to the effective masses entering the argument of the logarithm in $\hat{U}_{\text {eff }}$. It increases with temperature. This was one of the reasons why we have chosen the large- $N_{f}$-expansion. The original hope to completely avoid imaginary parts of $\hat{U}_{\text {eff }}$ in this scheme could not be confirmed by the results. The effective potential is still

plagued with imaginary parts for certain regions, where $\left|\sigma_{0,8}\right| \leq\left|\left\langle\sigma_{0,8}\right\rangle\right|$ and over the entire temperature range we have studied (up to $250[\mathrm{MeV}]$ and above). 
The large error bars on $\varepsilon$ and $s$ in the transition region may leave some doubts on the smooth nature of the conversion between the chiral symmetric and the chirally broken phase, in particular, because a smooth crossover in the condensates does not automatically imply a smooth change in $\varepsilon$ and $s$. Even if the transition would be of first order, and even if we use a loose bound on the latent heat, it is as small as 0.2 $\left[\mathrm{GeV} / \mathrm{fm}^{3}\right]$.

The ratio of critical to realistic current light quark masses has been estimated as $m_{u, d}^{\text {crit }} / m_{u, d} \sim 0.03 \pm 0.02$ in mean-field and as $m_{u, d}^{\text {crit }} / m_{u, d} \sim 0.26 \pm 0.08$ in large$N_{f}$. The large- $N_{f}$ result implies that realistic quark/meson masses lie less deeply in the crossover region than the mean-field result suggests. Due to the fluctuations which are effectively included in the large- $N_{f^{-}}$approximation the ratio in large- $N_{f}$ is about half of the lattice result. The average pseudoscalar octet masses are 126.4 $[\mathrm{MeV}]$ and $203[\mathrm{MeV}]$ for the realistic ratio of $\varepsilon_{0}^{\text {crit }} / \varepsilon_{8}^{\text {crit }}$ in mean-field and large- $N_{f}$, respectively. Note that an error of $30 \%$ in the critical quark masses blows up to an error of $55 \%$ in the critical meson masses, if the meson mass squares are linear in the current quark masses. Thus the critical values for the meson masses are not very conclusive.

In view of heavy-ion experiments one may conclude that there is still little hope for measurable experimental signatures of the crossover region, although the ratio $m_{u, d}^{c r i t} / m_{u, d}$ has become larger than the mean-field estimate. Quantities which are indirectly observable such as condensates, energy and entropy densities vary too smoothly during the phase conversion. Experimentalists should be warned, however, to accept these conclusions without care.

The tendency that $m_{u, d}^{\text {crit }} / m_{u, d}$ is larger in large- $N_{f}$ than in mean-field is plausible as an effect of the included fluctuations, but as we have seen this effect could not be confirmed for the $S U(3)$-symmetric or the $m_{s}=0$ case. The critical mass ratios there were even smaller in large- $N_{f}$ than in mean-field or at least of the same order of magnitude within the errors. This gives a hint that the fluctuations we include in our approximation are likely not the only important ones. In particular, it is not clear that they account for fluctuations which induce a renormalization of the quartic and cubic couplings in the Lagrangian.

Recently it has been raised by Gavin, Goksch and Pisarski 17 that the first order of the chiral transition may be mainly fluctuation induced. A fluctuation induced transition has been first discovered by Coleman and Weinberg [37]. It refers to the situation that a system with more than one relevant coupling can have 
a first order transition induced by quantum fluctuations. In an understanding of the renormalization group the fluctuation induced transition occurs due to so-called runaway RG trajectories (see e.g. [38]). Such an origin for a first order transition has been demonstrated in $4-\varepsilon$ dimensions for the $S U(N) \times S U(N)$ linear sigma model by Paterson [39], for more general symmetry groups $(O(N) \times O(M), U(N) \times U(M))$ by Pisarski and Stein [40]. In 4 dimensions Shen [38 has shown in a numerical simulation of a $U(N) \times U(N)$ symmetric scalar model that a fluctuation induced first order transition occurs in particular for large coupling $f_{2}$. For large and slowly varying $f_{2}, f_{1}$ runs fast from large to small values as a function of energy scale. The same tendency in an $S U(3) \times S U(3)$ linear sigma model would be compatible with a larger critical mass ratio $m_{u, d}^{c r i t} / m_{u, d}$. The larger mass ratio of the lattice simulation points into the same direction, since the lattice includes all fluctuations at once.

In our present approach it is difficult to disentangle the driving mechanism for the first order transition below the critical mass values. A further improvement by including subleading corrections in $1 / N_{f}$ or an $\varepsilon$-expansion in $d=4-\varepsilon$ dimensions should clarify, whether the ratio $m_{u, d}^{c r i t} / m_{u, d}$ does change to larger values. If the realistic finite quark masses lie in fact closer to the first order transition boundary than our results suggest, remnants of the first or second order transition may be more easily visible in experiments.

A second warning should be mentioned not to take the smooth crossover for guaranteed by the present approach. So far we have completely neglected the quarkand gluonic substructure. In particular the rearrangement of gluonic degrees of freedom has not been taken into account. The gluonic contribution to the change in entropy and energy densities may well accelerate the crossover process. Note that we have chosen the couplings in the sigma-model as temperature and energy scale independent over all temperatures up to $T_{c}$. In principle, the temperature and scale dependence of the couplings should be derived from QCD rather than being assumed. Less ambitious it may be derived from an effective model underlying the sigma-model and containing quark and gluonic degrees of freedom. For the critical mass ratio this offers at least a chance for being closer to 1 . Work in this direction is in progress.

\section{Acknowledgments}

We would like to thank H.-J. Pirner for useful discussions. 


\section{References}

[1] R.D. Pisarski and F. Wilczek, Phys. Rev. D29 (1984) 338.

[2] B. Svetitsky and L.G. Yaffe, Phys. Rev. D26 (1982) 963; Nucl. Phys. B210 [FS6] (1982) 423.

[3] J. Gasser and H. Leutwyler, Phys. Rep. 87 (1982) 77.

[4] P. Gerber and H. Leutwyler, Nucl. Phys. B321 (1989) 387.

[5] T.A. DeGrand and C.E. DeTar, Nucl. Phys. B225 [FS9] (1983) 590.

[6] T. Banks and A. Ukawa, Nucl. Phys. B225 [FS9] (1983) 145.

[7] F.R. Brown, F.P. Butler, H. Chen, N.H. Christ, Z. Dong, W. Schaffer, L. Unger, and A. Vaccarino, Phys. Rev. Lett. 65 (1990) 2491.

[8] G. Boyd, J. Fingberg, F. Karsch, L. Kärkkäinen and B. Petersson, Nucl. Phys. B376 (1992) 199.

[9] F. Karsch, Phys. Rev. D49 (1994) 3791.

[10] N.H. Christ, Nucl. Phys. B (Proc. Suppl.) 26 (1992) 217; Z. Dong and N.H. Christ, Nucl. Phys. B (Proc. Suppl.) 26 (1992) 314.

[11] H. Meyer-Ortmanns, H.J. Pirner and A. Patkós, Phys. Lett. B295 (1992) 255; Int. J. Mod. Phys. C3 (1992) 993.

[12] F. Wilczek, Int. J. Mod. Phys. A7 (1992) 3911; K. Rajagopal and F. Wilczek, Nucl. Phys. B379 (1993) 395.

[13] A. Bialas and R. Peschanski, Nucl. Phys. B273 (1986) 703; Nucl. Phys. B308 (1988) 857.

[14] J.-P. Blaizot and J.-Y. Ollitrault, in Quark-Gluon Plasma, ed. by R.C. Hwa (World Scientific, Singapore, 1990) p. 393.

[15] L. van Hove, Z. Phys. C27 (1985) 135.

[16] L.-H. Chan and R.W. Haymaker, Phys. Rev. D7 (1973) 415.

[17] S. Gavin, A. Goksch and R.D. Pisarski, Phys. Rev. D49 (1994) R3079.

[18] D. Metzger, H. Meyer-Ortmanns and H.-J. Pirner, Phys. Lett. B321 (1994) 66; Erratum, Phys. Lett. B328 (1994) 547. 
[19] S. Narison, QCD Spectral Sum Rules, Lecture Notes in Physics, Vol. 26 (World Scientific, Singapore, 1989) p. 184, 217.

[20] H. Goldberg, Phys. Lett. B131 (1983) 133.

[21] Z. Frei and A. Patkós, Phys. Lett. 247B (1990) 381.

[22] S. Coleman, R. Jackiw and H.D. Politzer, Phys. Rev. D10 (1974) 2491.

[23] L. Dolan and R. Jackiw, Phys. Rev. D9 (1974) 3320.

[24] H.E. Haber and H.A. Weldon, J. Math. Phys. 23 (1982) 1852.

[25] M. Abramovitz and I.A. Stegun, eds., Handbook of Mathematical Functions (National Bureau of Standards, Washington, 1964) p. 375.

[26] W.H. Press, B.P. Flannery, S.A. Teukolsky, W.T. Vetterling, eds., Numerical Recipies (Cambridge University Press, Cambridge, 1986) p. 170.

[27] J. Cleymans, A. Kocić and M. D. Scadron, Phys. Rev. D39 (1989) 323.

[28] K. Kajantie, Int. J. Mod. Phys. C3 (1992) 1137.

[29] H. Leutwyler, Phys. Lett. B284 (1992) 106.

[30] M. Lutz, S. Klimt and W. Weise, Nucl. Phys. A542 (1992) 521.

[31] V. Jain, Nucl. Phys. B394 (1993) 707.

[32] T. Hatsuda and T. Kunihiro, Phys. Lett. B198 (1987) 126; T. Hatsuda, in Proc. Quark Matter '91, Nucl. Phys. A544 (1992) 27c.

[33] J. Engels, J. Fingberg, F. Karsch, D. Miller and M. Weber, Phys. Lett. B252 (1990) 625.

[34] F.R. Brown, N.H. Christ, Y. Deng, M. Gao and T.J. Woch, Phys. Rev. Lett. 61 (1988) 2058; Y. Iwasaki, K. Kanaya, T. Yoshié, T. Hoshino, T. Shirakawa, Y. Oyanagi, S. Ichii and T. Kawai, Phys. Rev. Lett. 67 (1991) 3343.

[35] J. Kogut and D.K. Sinclair, Phys. Rev. Lett. 60 (1988) 1250.

[36] F. Karsch, in Proc. QCD 20 Years Later, ed. by P.M. Zerwas and H.A. Kastrup, Vol. 2 (World Scientific, Singapore, 1993) p. 717.

[37] S. Coleman and E. Weinberg, Phys. Rev. D7 (1973) 1888. 
[38] Y. Shen, Phys. Lett. B315 (1993) 146.

[39] A.J. Paterson, Nucl. Phys. B190 (1981) 188.

[40] R.D. Pisarski and D.L. Stein, Phys. Rev. B23 (1981) 3549; J. Phys. A14 (1981) 3341. 
This figure "fig1-1.png" is available in "png" format from: http://arxiv.org/ps/hep-ph/9409430v1 
This figure "fig2-1.png" is available in "png" format from: http://arxiv.org/ps/hep-ph/9409430v1 
This figure "fig3-1.png" is available in "png" format from: http://arxiv.org/ps/hep-ph/9409430v1 
This figure "fig4-1.png" is available in "png" format from: http://arxiv.org/ps/hep-ph/9409430v1 
This figure "fig1-2.png" is available in "png" format from: http://arxiv.org/ps/hep-ph/9409430v1 
This figure "fig2-2.png" is available in "png" format from: http://arxiv.org/ps/hep-ph/9409430v1 
This figure "fig3-2.png" is available in "png" format from: http://arxiv.org/ps/hep-ph/9409430v1 
This figure "fig4-2.png" is available in "png" format from: http://arxiv.org/ps/hep-ph/9409430v1 
This figure "fig2-3.png" is available in "png" format from: http://arxiv.org/ps/hep-ph/9409430v1 
This figure "fig3-3.png" is available in "png" format from: http://arxiv.org/ps/hep-ph/9409430v1 
This figure "fig4-3.png" is available in "png" format from: http://arxiv.org/ps/hep-ph/9409430v1 